\title{
Adaptive Multivariable Integral TSMC of a Hypersonic Gliding Vehicle with Actuator Faults and Model Uncertainties
}

\author{
Peng Li, Xiang Yu, Senior Member, IEEE, Youmin Zhang, Senior Member, IEEE, and Xiaoyan Peng
}

\begin{abstract}
This paper presents a fault-tolerant control (FTC) strategy for a hypersonic gliding vehicle (HGV) subject to actuator malfunctions and model uncertainties. The controloriented model of the HGV is estabilished according to the HGV kinematic and aerodynamic models. A composite-loop design for HGV FTC under actuator faults is subsequently developed, where newly developed multivariable integral terminal sliding mode control (TSMC) and adaptive techniques are integrated. The multivariable integral TSMC is capable of ensuring the finitetime stability of the closed-loop system in the presence of actuator malfunctions and model uncertainties, while the adaptive laws are employed to tune the control parameters in response to the HGV status. Simulation studies based on a six degree-of-freedom (DOF) nonlinear model of the HGV are illustrated to highlight the effectiveness of the developed FTC scheme.
\end{abstract}

Index Terms-Actuator faults, hypersonic gliding vehicle, faulttolerant control, multivariable integral TSMC, control-oriented model, composite-loop design.

\section{INTRODUCTION}

Hypersonic gliding vehicle ( $\mathrm{HGV}$ ) is designed with the aerodynamic configuration of high lift-to-drag ratio, which can be launched into the sub-orbital trajectory either by a booster rocket or a reusable launch vehicle. Without any power, an HGV that can operate in near space with a speed of more than Mach 5, possesses a capacity for "extreme maneuvers". Benefited from the rapid response and flexible maneuverability, HGVs are recognized as a viable option of long-range delivery, remote rapid strike, and power projection.

One of the major problems is that large uncertainties and perturbations are inherent to the HGV model [1]. The HGV unique characteristics pose a severe challenge for the design of flight control systems. The vast majority of contributions focus on control methodologies, including adaptive control [2, 3], back-stepping and dynamic inversion control [4], linear

This work was supported in part the National Natural Science Foundation of China under Grants 61403407, 51575167, 61573282 and 61603130, in part by NSERC, in part by the Natural Science Foundation of Shaanxi Province under Grant 2015JZ020, in part by the Open Project Program of the State Key Laboratory of High Performance Computing under Grants 201613-02.

Peng Li (lipeng_2010@163.com) is with the College of Mechatronics Engineering and Automation, National University of Defense Technology, Changsha, 410073, China, and also with the State Key Laboratory of High Performance Computing in the same university.

Xiang Yu (Corresponding Author: xiangyu1110@gmail.com) and Youmin Zhang (youmin.zhang@concordia.ca) are with the Department of Mechanical, Industrial and Aerospace Engineering, Concordia University, Montreal, Quebec, H3G 1M8, Canada.

Xiaoyan Peng (xypeng.hnu@gmail.com) is with the College of Mechanical and Vehicle Engineering, Hunan University, Changsha, 410082, China. parameter varying control [5], sliding mode control (SMC) [6], model predictive control [7], robust control [8], and adaptive continuous higher order sliding mode control (HOSMC) [9].

Requirements that drive the desire for HGVs include reliability and maintainability. By contrast, increasing complexity and automation make an HGV more vulnerable to component/system malfunctions across the flight envelope. Faulttolerant control (FTC), which can maintain the safety of a post-fault plant, has drawn considerable attention in aerospace engineering systems $[10,11]$. As a consequence, the safety demand for hypersonic flights has spurred an interest in the FTC design. Takagi-Sugeno (T-S) fuzzy system and adaptive control have been exploited in the FTC design of hypersonic flight vehicles $[12,13]$. Within the scheme [14], a group of local FTCs are synthesized in response to various faults, whilst Youla parameterizations are constructed to tolerate arbitrary switching actions among different fault modes of a hypersonic vehicle. A model predictive based FTC is investigated in [15], by which the reshaped reference can be tracked in the actuator faulty condition.

Two-loop controllers based on SMC are deployed to constitute an active FTC scheme for hypersonic vehicle attitude control. The resulting FTC system can guarantee the asymptotic output tracking in spite of actuator faults [16]. An FTC system on the basis of back-stepping and sliding mode technologies is applied for a hypersonic aircraft. The studied controller can guarantee the safety of the handicapped aircraft and the globally asymptotic tracking performance [17]. Recent effort [18] attempts to design an FTC system with combination of conventional SMC and nonlinear disturbance observer. Note that the actuator amplitude constraints and faults are taken into account over the FTC design phase. Furthermore, terminal SMC (TSMC) techniques are adopted to advance the state of the art of FTC. TSMC can not only possess strong robustness on uncertain dynamics similar to linear SMC, but also guarantee the finite-time convergence of tracking error [19-21]. Two dynamic TSMCs are designed with respect to the inner and outer loops, handling actuator faults of a hypersonic vehicle [22]. An FTC strategy is determined by resorting to TSMC approach, ensuring that velocity and altitude track the reference signals in finite time after occurrence of actuator malfunctions [23]. The work in [24], which develops a passive FTC based on TSMC technique, focuses on enhancing the convergence rate.

Despite that previous studies have gained various degrees of success in addressing HGV FTC issues, there still exist some 
challenges. 1) The amount of fault recovery time, which solely relies on the operating condition and the fault characteristics, is very limited for safety-critical plants $[10,11]$. More particularly, the safety restrictions imposed on HGV inputs and outputs may be violated, if faults cannot be accommodated within the allowable amount of time. From this perspective, more emphasis on the HGV FTC design with a timely manner needs to be placed. 2) In terms of the time-scale separation principle, it is common practice to approach the HGV FTC problem by independent design of fast inner-loop and slow outer-loop dynamics $[25,26]$. In the outer-loop, the angular rate profiles, which are regarded as virtual control signals to the inner-loop, are produced by the kinematics equation of angular motion and the SMC. With respect to the inner loop, another SMC is synthesized such that the commanded angular rate profiles are tracked. Roll, pitch, and yaw torque commands generated by the inner-loop are then allocated into control surface deflection commands. However, how to guarantee the finite-time stability of the overall system is an open issue. 3) TSMC is exploited for stabilizing the HGV subject to faults and uncertainties. Nonetheless, in the most of resulting TSMC approaches, a multi-input control problem with $m$ control inputs is transformed into a decoupled problem involving $m$ single input control structures. This type of approach may not be effective due to strong couplings in HGV aerodynamics.

In an attempt to tackle the above-mentioned issues, a TSMC based FTC design approach is proposed against HGV actuator faults and model uncertainties, with particular attention devoted to achieving multivariable design in a composite-loop. The major contributions are briefly outlined as follows.

1) Due to lack of wind tunnel facility and flight test experiments, a partial knowledge of the aerodynamic derivatives of hypersonic vehicles is present. The control input matrix in any $\mathrm{HGV}$ is composed of control moment coefficients, which are extremely difficult to accurately obtain in comparison of conventional aircraft. Hence, multiplicative uncertainty exists in the $\mathrm{HGV}$ control input matrix, inducing a great challenge of control design especially in the event of actuator faults. In this study, the cases of $\mathrm{HGV}$ actuator malfunctions and multiplicative uncertainty in control input matrix are simultaneously considered at the FTC design stage. To the best of the authors' knowledge, there are few papers focusing on this aspect.

2) In most of the existing literature, control design of hypersonic vehicles is divided into the inner loop and outer loop design (named dual-loop design) based on timescale separation principle. However, this type of design cannot ensure the stability of the overall closed-loop system. This study establishes a control-oriented model by integrating the $\mathrm{HGV}$ attitude kinematic and dynamic equations. Subsequently, a composite-loop design for $\mathrm{HGV}$ attitude tracking control under actuator faults is developed. The finite-time stability of the closed-loop system can be guaranteed from a theoretical perspective.

3) A finite-time multivariable TSMC approach based on homogeneity is exploited in the FTC design. With consideration of $\mathrm{HGV}$ actuator malfunctions and model uncertainties, a novel integral terminal sliding mode surface is established by introducing the fractional power integral terms. The resulting FTC can ensure the finitetime stability of the $\mathrm{HGV}$, when actuator faults and model uncertainties exist. Moreover, the multivariable integral TSMC formed by vector expression, which is driven directly from the sliding mode reachability condition, is incorporated in the HGV FTC design. This feature is of significance in the sense that the strong couplings are inherent to $\mathrm{HGV}$ aerodynamics.

The rest of this paper is organized as follows. The concepts of finite-time stable system and homogeneity are described in Section II. The HGV aerodynamics, actuator fault model, and problem statement are given in Section III. The controloriented model is presented in Section IV. The HGV FTC scheme is proposed against actuator faults and model uncertainties in Section V. In Section VI, the performance of the developed FTC is evaluated through simulations of a full nonlinear model of the HGV dynamics. Section VII includes a discussion of the conclusions.

\section{PRELIMINARIES}

A brief description of finite-time stability and homogeneity is presented, serving as a foundation of the HGV FTC design. Consider the system:

$$
\dot{\boldsymbol{\xi}}=\boldsymbol{f}(\boldsymbol{\xi}), \boldsymbol{f}(\mathbf{0})=\mathbf{0}, \boldsymbol{\xi} \in R^{n}, \boldsymbol{\xi}(\mathbf{0})=\boldsymbol{x}_{0},
$$

where $f: D \rightarrow R^{n}$ is continuous on an open neighborhood $D$ of the origin $\boldsymbol{\xi}=0$. The equilibrium $\boldsymbol{\xi}=\mathbf{0}$ of (1) is finitetime convergent if there are an open neighborhood $U \subseteq D$ of the origin and a function $T_{\boldsymbol{\xi}}: U \backslash\{\mathbf{0}\} \rightarrow(0, \infty)$, such that $\forall \boldsymbol{\xi}_{0} \in U$, the solution trajectories $\boldsymbol{\xi}\left(t, \boldsymbol{\xi}_{0}\right)$ of (1) starting from the initial point $\boldsymbol{\xi}_{0} \in U \backslash\{0\}$ is well-defined and unique in forward time for $t \in\left[0, T_{\boldsymbol{\xi}}\left(\boldsymbol{\xi}_{0}\right)\right)$, and $\lim _{t \rightarrow T_{\boldsymbol{\xi}}\left(\boldsymbol{\xi}_{0}\right)} \boldsymbol{\xi}\left(t, \boldsymbol{\xi}_{0}\right)=\mathbf{0}$. Here $T_{\boldsymbol{\xi}}\left(\boldsymbol{\xi}_{0}\right)$ is called the settling time (of the initial state $\boldsymbol{\xi}_{0}$ ). The equilibrium of (1) is finite-time stable if it is Lyapunov stable and finite-time convergent. When $U=D=R^{n}$, then the origin is in globally finite-time stable equilibrium.

Definition 1: Let dilation $\left(r_{1}, \cdots, r_{n}\right) \in R^{n}$ with $r_{i}>0$, $i=1, \cdots, n$. Let $\boldsymbol{f}(\boldsymbol{\xi})=\left[f_{1}(\boldsymbol{\xi}), \cdots, f_{n}(\boldsymbol{\xi})\right]^{T}$ be a continuous vector field. $\boldsymbol{f}(\boldsymbol{\xi})$ is recognized to be homogeneous of degree $d \in R$ with respect to dilation $\left(r_{1}, \cdots, r_{n}\right)$ if, for any given $\varepsilon>0$,

$$
\begin{array}{r}
f_{i}\left(\varepsilon^{r_{1}} \xi_{1}, \cdots, \varepsilon^{r_{n}} \xi_{n}\right)=\varepsilon^{d+r_{i}} f_{i}(\boldsymbol{\xi}), \\
i=1, \ldots n, \forall \xi \in R^{n} .
\end{array}
$$

System (1) is said to be homogeneous if $\boldsymbol{f}(\boldsymbol{\xi})$ is homogeneous.

Lemma 1 [27]: The continuous system (1) is named globally finite-time stable if it is globally asymptotical stable and locally homogeneous of degree $d<0$.

\section{Mathematical Model of A HGV}

\section{A. Nonlinear HGV Model}

The HGV model is based on the assumption of a rigid vehicle structure, a flat, non-rotating Earth and uniform gravitational field. In the following, the kinematic model and 
dynamic model of a HGV are described, respectively. The inertial position coordinates are described as:

$$
\left\{\begin{array}{l}
\dot{x}=V \cos \gamma \cos \chi \\
\dot{y}=V \cos \gamma \sin \chi \\
\dot{z}=-V \sin \gamma
\end{array},\right.
$$

where $x, y$, and $z$ represent the positions with respect to $x-, y$-, and $z$-directions of the Earth-fixed reference frame, respectively. $V$ stands for the total velocity, $\gamma$ and $\chi$ denote the flight-path angle and the heading angle, respectively.

The force equations are described as:

$$
\left\{\begin{array}{l}
\dot{V}=-g \sin \gamma-\frac{Q S_{r} C_{D}}{m} \\
\dot{\chi}=\frac{Q S_{r}}{m V \cos \gamma}\left(C_{L} \sin \mu+C_{Y} \cos \mu\right) \\
\dot{\gamma}=-\frac{g}{V} \cos \gamma+\frac{Q S_{r}}{m V}\left(C_{L} \cos \mu-C_{Y} \sin \mu\right)
\end{array},\right.
$$

where $\mu$ is the bank angle, $g$ is the gravitational constant, $Q$ is the dynamic pressure, $S_{r}$ is the reference area, $m$ is the HGV mass, $C_{L}, C_{D}$, and $C_{Y}$ are the aerodynamic coefficients with respect to lift, drag, and side force, respectively.

The model of attitude is written as:

$$
\left\{\begin{array}{rl}
\dot{\mu}= & \sec \beta(p \cos \alpha+r \sin \alpha) \\
& +\frac{Q S_{r} C_{L}}{m V}(\tan \gamma \sin \mu+\tan \beta) \\
& +\frac{Q S_{r} C_{Y}}{m V} \tan \gamma \cos \mu-\frac{g}{V} \cos \gamma \cos \mu \tan \beta \\
\dot{\alpha}= & q-\tan \beta(p \cos \alpha+r \sin \alpha) \\
& +\frac{1}{m V \cos \beta}\left(m g \cos \gamma \cos \mu-Q S_{r} C_{L}\right) \\
\dot{\beta}= & -r \cos \alpha+p \sin \alpha \\
& +\frac{1}{m V}\left(Q S_{r} C_{Y}+m g \cos \gamma \sin \mu\right)
\end{array},\right.
$$

where $\alpha$ and $\beta$ denote the angle of attack (AOA) and sideslip angle, respectively.

The model of angular velocities is given as:

$$
\left\{\begin{array}{l}
\dot{p}=\frac{l_{A}+\left(I_{y y}-I_{z z}\right) q r}{I_{x x}} \\
\dot{q}=\frac{m_{A}+\left(I_{z z}-I_{x x}\right) p r}{I_{y y}} \\
\dot{r}=\frac{n_{A}+\left(I_{x x}-I_{y y}\right) p q}{I_{z z}}
\end{array}\right.
$$

where $p, q$, and $r$ are roll, pitch, and yaw angular rates, respectively. $l_{A}, m_{A}$, and $n_{A}$ denote the roll, pitch, and yaw moments, while $I_{x x}, I_{y y}$, and $I_{z z}$ represent the moments of inertia.

The aerodynamics forces $L, D$, and $Y$ are represented as:

$$
\left\{\begin{array}{l}
L=Q S_{r} C_{L} \\
D=Q S_{r} C_{D} \\
Y=Q S_{r} C_{Y}
\end{array}\right.
$$

where $C_{L}=C_{L, \text { clean }}+C_{L, \delta_{a}} \delta_{a}+C_{L, \delta_{e}} \delta_{e}, C_{D}=C_{D, \text { clean }}+$ $C_{D, \delta_{a}} \delta_{a}+C_{D, \delta_{e}} \delta_{e}+C_{D, \delta_{r}} \delta_{r}$, and $C_{Y}=C_{Y, \beta}+C_{Y, \delta_{a}} \delta_{a}+$ $C_{Y, \delta_{e}} \delta_{e}+C_{Y, \delta_{r}} \delta_{r} . \delta_{a}, \delta_{e}$, and $\delta_{r}$ are the so-called control deflections of the aileron, elevator, and rudder, respectively.

The rolling, pitching, and yawing moments are:

$$
\left\{\begin{array}{l}
l_{A}=Q b S_{r} C_{l} \\
m_{A}=Q c S_{r} C_{m}-x_{c g}(-D \sin \alpha-L \cos \alpha) \\
n_{A}=Q b S_{r} C_{n}+x_{c g} Y
\end{array}\right.
$$

where $b$ is the span of the $\mathrm{HGV}, c$ is the mean aerodynamic chord, $x_{c g}$ is the distance between the centroid and reference moment along $x$ body-axis. The corresponding coefficients are calculated as: $C_{l}=C_{l, \beta} \beta+C_{l, \delta_{a}} \delta_{a}+C_{l, \delta_{e}} \delta_{e}+C_{l, \delta_{r}} \delta_{r}+$
$C_{l, r} \frac{r b}{2 V}+C_{l, p} \frac{p b}{2 V}, C_{m}=C_{m, \text { clean }}+C_{m, \delta_{a}} \delta_{a}+C_{m, \delta_{e}} \delta_{e}+$ $C_{m, \delta_{r}} \delta_{r}+C_{m, q} \frac{q c}{2 V}$, and $C_{n}=C_{n, \beta} \beta+C_{n, \delta_{a}} \delta_{a}+C_{n, \delta_{e}} \delta_{e}+$ $C_{n, \delta_{r}} \delta_{r}+C_{n, p} \frac{p b}{2 V}+C_{n, r} \frac{r b}{2 V}$.

\section{B. Actuator Fault Model}

When actuation systems work under a normal condition, appropriate aerodynamic forces and moments are produced. The required HGV maneuver can be thereby accomplished with a baseline/nominal controller. If the HGV encounters actuator malfunctions, the nominal controller's attempts to maintain the expected maneuver may be futile and the flight safety can be jeopardized [28, 29]. Gain fault and bias fault are the faults commonly appearing on flight actuators. The actuator fault model is generally formed as:

$$
\boldsymbol{u}_{F}=\boldsymbol{\Lambda} \boldsymbol{u}+\boldsymbol{\rho},
$$

where $\boldsymbol{\Lambda}=\operatorname{diag}\left\{\lambda_{1}, \lambda_{2}, \lambda_{3}\right\}$ represents the gain fault, $\boldsymbol{\rho}=$ $\left[\rho_{1}, \rho_{2}, \rho_{3}\right]^{T}$ denotes the bias fault, and $\boldsymbol{u}=\left[\delta_{a}, \delta_{e}, \delta_{r}\right]^{T}$.

Remark 1: It is reported in [28] that the leakage of hydraulic fluid can be the root cause of the degradation of the actuator effectiveness. Therefore, $\boldsymbol{\Lambda}=\operatorname{diag}\left\{\lambda_{1}, \lambda_{2}, \lambda_{3}\right\}$ in Eq. (9) is used to describe the effectiveness of the HGV actuators, where $0<\lambda_{1}, \lambda_{2}, \lambda_{3} \leq 1$. In addition, the sensor fault in an actuator system can result in the actuator bias faults. To be more specific, if the amplitude sensor encounters a bias fault, the measured amplitude is the actual amplitude plus the bias value. As a consequence, the sensed amplitude is forced to be equal to the referenced signal. However, the actual value of the actuator amplitude is deviated from the expected value. Hence, $\boldsymbol{\rho}=\left[\rho_{1}, \rho_{2}, \rho_{3}\right]^{T}$ is adopted in Eq. (9) to describe the bias faults of the aileron, elevator, and rudder, respectively.

\section{Problem Statement}

The purpose is to develop an FTC scheme based on adaptive multivariable integral TSMC such that:

1) The deleterious effects of HGV actuator faults can be compensated within a finite amount of time, thus:

$$
\lim _{t \rightarrow t_{f}}\left|\mu-\mu_{d}\right|=0, \lim _{t \rightarrow t_{f}}\left|\alpha-\alpha_{d}\right|=0, \lim _{t \rightarrow t_{f}}\left|\beta-\beta_{d}\right|=0
$$

where $t_{f}$ is the finite time, $\mu_{d}, \alpha_{d}$, and $\beta_{d}$ correspond to the reference commands of the bank angle, AOA, and sideslip angle, respectively;

2) A composite-loop design for HGV attitude tracking control under actuator faults can be achieved, without the need of dividing the HGV dynamics into the innerloop and outer-loop;

3) Multivariable design can be integrated into the FTC.

\section{Control-Oriented Model}

This section details the establishment of the control-oriented model, which provides a basis of the composite-loop design of the HGV FTC. 
$\boldsymbol{g}_{2}=\left[\begin{array}{ccc}\frac{Q b S_{r} C_{l, \delta a}}{I_{x}} & \frac{Q b S_{r} C_{l, \delta e}}{I_{x}} & \frac{Q b S_{r} C_{l, \delta r}}{I_{x x}} \\ \frac{Q c S_{r} C_{m, \delta a}+Q x_{c g} S_{r}\left(C_{D, \delta a} \sin \alpha+C_{L, \delta a} \cos \alpha\right)}{I_{y y}} & \frac{Q c S_{r} C_{m, \delta e}+Q x_{c g} S_{r}\left(C_{D, \delta e} \sin \alpha+C_{L, \delta e} \cos \alpha\right)}{I_{y y}} & \frac{Q c S_{r} C_{m, \delta r}+Q x_{c g} S_{r} C_{D, \delta r} \sin \alpha}{I_{y y}} \\ \frac{Q b S_{r} C_{n, \delta a}+Q x_{c g} S_{r} C_{Y, \delta a}}{I_{z z}} & \frac{Q b S_{r} C_{n, \delta e}+Q x_{c g} S_{r} C_{Y, \delta e}}{I_{z z}} & \frac{Q b S_{r} C_{n, \delta r}+Q x_{c g} S_{r} C_{Y, \delta r}}{I_{z z}}\end{array}\right]$

For the fault-tolerant attitude control system of the HGV, define $\boldsymbol{x}_{1}=[\mu, \alpha, \beta]^{T}$ and $\boldsymbol{x}_{2}=[p, q, r]^{T}$. In accordance with Eq. (5), one can obtain:

$$
\left\{\begin{array}{l}
\dot{\mu}=\sec \beta(p \cos \alpha+r \sin \alpha)+f_{\mu} \\
\dot{\alpha}=q-\tan \beta(p \cos \alpha+r \sin \alpha)+f_{\alpha} \\
\dot{\beta}=-r \cos \alpha+p \sin \alpha+f_{\beta}
\end{array}\right.
$$

where

$$
\left\{\begin{aligned}
f_{\mu}= & \frac{Q S_{r} C_{L}}{m V}(\tan \gamma \sin \mu+\tan \beta) \\
& +\frac{Q S_{r} C_{Y}}{m V} \tan \gamma \cos \mu-\frac{g}{V} \cos \gamma \cos \mu \tan \beta \\
f_{\alpha}= & \frac{1}{m V \cos \beta}\left(m g \cos \gamma \cos \mu-Q S_{r} C_{L}\right) \\
f_{\beta}= & \frac{1}{m V}\left(Q S_{r} C_{Y} \cos \beta+m g \cos \gamma \sin \mu\right)
\end{aligned}\right.
$$

By recalling the definitions $\boldsymbol{x}_{1}=[\mu, \alpha, \beta]^{T}$ and $\boldsymbol{x}_{2}=$ $[p, q, r]^{T}$, Eqs. (11)-(12) can be therefore expressed as:

$$
\dot{\boldsymbol{x}}_{1}=\boldsymbol{f}_{1}+\boldsymbol{g}_{1} \boldsymbol{x}_{2}
$$

where $\boldsymbol{f}_{1}=\left[f_{\mu}, f_{\alpha}, f_{\beta}\right]^{T}$ and

$$
\boldsymbol{g}_{1}=\left[\begin{array}{ccc}
\sec \beta \cos \alpha & 0 & \sec \beta \sin \alpha \\
-\tan \beta \cos \alpha & 1 & -\tan \beta \sin \alpha \\
\sin \alpha & 0 & -\cos \alpha
\end{array}\right] \text {. }
$$

Moreover, combining the angular rate dynamics of Eq. (6) and aerodynamic moments of Eq. (8) gives:

$$
\begin{aligned}
\dot{p}= & \left(Q b S_{r}\left(C_{l, \beta} \beta+C_{l, r}\left(\frac{r b}{2 V}\right)+C_{l, p}\left(\frac{p b}{2 V}\right)\right) / I_{x x}\right. \\
& +\left(\left(I_{y y}-I_{z z}\right) q r\right) / I_{x x} \\
& +\left(Q b S_{r}\left(C_{l, \delta a} \delta_{a}+C_{l, \delta e} \delta_{e}+C_{l, \delta r} \delta_{r}\right)\right) / I_{x x} .
\end{aligned}
$$

By defining $f_{p}=\left(Q b S_{r}\left(C_{l, \beta} \beta+C_{l, r}\left(\frac{r b}{2 V}\right)+C_{l, p}\left(\frac{p b}{2 V}\right)\right) / I_{x x}+\right.$ $\left(\left(I_{y y}-I_{z z}\right) q r\right) / I_{x x}$, Eq. (15) can be recast in the form:

$$
\begin{aligned}
\dot{p}= & f_{p}+\left[\frac{Q b S_{r} C_{l, \delta a}}{I_{x x}}, \frac{Q b S_{r} C_{l, \delta e}}{I_{x x}}, \frac{Q b S_{r} C_{l, \delta r}}{I_{x x}}\right] \\
& \cdot\left[\begin{array}{lll}
\delta_{a} & \delta_{e} & \delta_{r}
\end{array}\right]^{T} .
\end{aligned}
$$

Similarly, the pitch angular rate dynamics is represented as:

$$
\begin{aligned}
\dot{q}=( & Q c S_{r}\left(C_{m, \text { clean }}+C_{m, q}\left(\frac{q c}{2 V}\right)\right) / I_{y y} \\
& \left.+Q x_{c g} S_{r}\left(C_{D, \text { clean }} \sin \alpha+C_{L, \text { clean }} \cos \alpha\right)\right) / I_{y y} \\
& +\left(Q c S_{r}\left(C_{m, \delta a} \delta_{a}+C_{m, \delta e} \delta_{e}+C_{m, \delta r} \delta_{r}\right)\right) / I_{y y} \\
& +\left(Q x_{c g} S_{r}\left(\left(C_{D, \delta a} \sin \alpha+C_{L, \delta a} \cos \alpha\right) \delta_{a}\right)\right) / I_{y y} \\
& +\left(Q x_{c g} S_{r}\left(\left(C_{D, \delta e} \sin \alpha+C_{L, \delta a} \cos \alpha\right) \delta_{e}\right)\right) / I_{y y} \\
& +\left(Q x_{c g} S_{r}\left(C_{D, \delta r} \sin \alpha \delta_{r}\right)\right) / I_{y y} \\
& +\left(\left(I_{z z}-I_{x x}\right) p r\right) / I_{y y} .
\end{aligned}
$$

As long as letting $f_{q}=\left(Q c S_{r}\left(C_{m, \text { clean }}+C_{m q}\left(\frac{q c}{2 V}\right)\right)\right) / I_{y y}+$ $\left(Q x_{c g} S_{r}\left(C_{D, \text { clean }} \sin \alpha+C_{L, \text { clean }} \cos \alpha\right)\right) / I_{y y}$

$\left(\left(I_{z z}-I_{x x}\right) p r\right) / I_{y y}$, Eq. (17) can be simplified as:

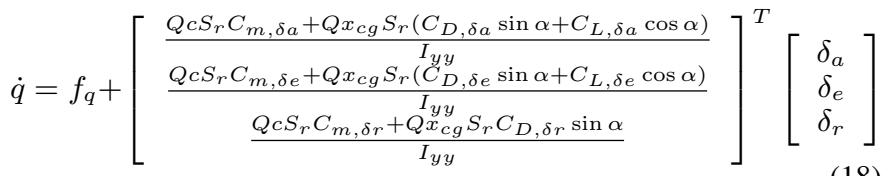

The yaw angular rate dynamics is described as:

$$
\dot{r}=f_{r}+\left[\begin{array}{c}
\frac{Q b S_{r} C_{n, \delta a}+Q x_{c g} S_{r} C_{Y, \delta a}}{I_{z} x_{c g} S_{r} C_{Y, \delta e}} \\
\frac{Q b S_{r} C_{n, \delta e}+I_{z}}{Q b S_{r} C_{n, \delta r}+x_{c g} S_{r} C_{Y, \delta r}} \\
I_{z z}
\end{array}\right]^{T}\left[\begin{array}{l}
\delta_{a} \\
\delta_{e} \\
\delta_{r}
\end{array}\right],
$$

where $f_{r}=Q b S_{r}\left(C_{n, \beta} \beta+C_{n p}\left(\frac{p b}{2 V}\right)+C_{n r}\left(\frac{r b}{2 V}\right)\right) / I_{z z}+$ $\left(Q x_{c g} S_{r} C_{Y, \beta} \beta+\left(I_{x x}-I_{y y}\right) p q\right) / I_{z z}$.

According to Eqs. (16), (18), and (19), one can obtain:

$$
\dot{\boldsymbol{x}}_{2}=\boldsymbol{f}_{2}+\boldsymbol{g}_{2} \boldsymbol{u}
$$

where $\boldsymbol{f}_{2}=\left[f_{p}, f_{q}, f_{r}\right]^{T}, \boldsymbol{u}=\left[\delta_{a}, \delta_{e}, \delta_{r}\right]^{T}$, and $\boldsymbol{g}_{2}$ is shown at the top of this page.

Combining Eqs. (13) and (20) gives:

$$
\left\{\begin{array}{l}
\dot{\boldsymbol{x}}_{1}=\boldsymbol{f}_{1}+\boldsymbol{g}_{1} \boldsymbol{x}_{2} \\
\dot{\boldsymbol{x}}_{2}=\boldsymbol{f}_{2}+\boldsymbol{g}_{2} \boldsymbol{u}
\end{array}\right.
$$

Differentiating Eq. (13) and recalling Eq. (20) achieve:

$$
\ddot{\boldsymbol{x}}_{1}=\dot{\boldsymbol{f}}_{1}+\dot{\boldsymbol{g}}_{1} \boldsymbol{x}_{2}+\boldsymbol{g}_{1} \boldsymbol{f}_{2}+\boldsymbol{g}_{1} \boldsymbol{g}_{2} \boldsymbol{u} .
$$

The control-oriented model with actuator anomalies and model uncertainties is built as follows. Firstly, Eq. (22) can be expressed as:

$$
\ddot{\boldsymbol{x}}_{1}=\dot{\boldsymbol{f}}_{1}+\boldsymbol{F}\left(\boldsymbol{x}_{1}, \boldsymbol{x}_{2}\right)+\boldsymbol{G}\left(\boldsymbol{x}_{1}, \boldsymbol{x}_{2}\right) \boldsymbol{u},
$$

where $\boldsymbol{F}\left(\boldsymbol{x}_{1}, \boldsymbol{x}_{2}\right)=\dot{\boldsymbol{g}}_{1} \boldsymbol{x}_{2}+\boldsymbol{g}_{1} \boldsymbol{f}_{2}$ and $\boldsymbol{G}\left(\boldsymbol{x}_{1}, \boldsymbol{x}_{2}\right)=\boldsymbol{g}_{1} \boldsymbol{g}_{2}$. In addition, $\boldsymbol{F}\left(\boldsymbol{x}_{1}, \boldsymbol{x}_{2}\right)$ contains two terms:

$$
\boldsymbol{F}=\boldsymbol{F}_{n}+\boldsymbol{\Delta}_{F},
$$

where $\boldsymbol{F}_{n}$ and $\boldsymbol{\Delta}_{F}$ denote the nominal portion and the uncertain portion of $\boldsymbol{F}$, respectively. $\boldsymbol{G}\left(\boldsymbol{x}_{1}, \boldsymbol{x}_{2}\right)$ can be specified in a manner similar to $\boldsymbol{F}\left(\boldsymbol{x}_{1}, \boldsymbol{x}_{2}\right)$ :

$$
\boldsymbol{G}=\boldsymbol{G}_{n}+\boldsymbol{\Delta}_{G}
$$

The nominal term of $\boldsymbol{G}$ is $\boldsymbol{G}_{n}$, which solely relies on the known portions of $\boldsymbol{g}_{1}$ and $\boldsymbol{g}_{2}$. With respect to the studied HGV, $\operatorname{det}\left(g_{1}\right)=-\sec \beta$. One can obtain that $\boldsymbol{g}_{1}$ is invertible if $\beta$ does not equal to $\pm \pi / 2$. Focusing on the known portion of $\boldsymbol{g}_{2}$, it can be regarded as control allocation matrix which is invertible in $\mathrm{HGV}$ flight envelopes. Therefore, the nominal portion $\boldsymbol{G}_{n}$ is invertible.

Consequently, Eq. (23) can be further written as:

$$
\ddot{\boldsymbol{x}}_{1}=\dot{\boldsymbol{f}}_{1}+\boldsymbol{F}_{n}+\boldsymbol{\Delta}_{F}+\left(\boldsymbol{G}_{n}+\boldsymbol{\Delta}_{G}\right) \boldsymbol{u} .
$$

By accounting for the gain and the bias faults in actuators as Eq. (9), one can render:

$$
\begin{aligned}
\ddot{\boldsymbol{x}}_{1}= & \dot{\boldsymbol{f}}_{1}+\boldsymbol{F}_{n}+\boldsymbol{\Delta}_{F}+\left(\boldsymbol{G}_{n}+\boldsymbol{\Delta}_{G}\right)(\boldsymbol{\Lambda} \boldsymbol{u}+\boldsymbol{\rho}) \\
= & \dot{\boldsymbol{f}}_{1}+\boldsymbol{F}_{n}+\boldsymbol{\Delta}_{F}+\left(\boldsymbol{G}_{n}+\boldsymbol{\Delta}_{G}\right) \boldsymbol{\rho} \\
& +\left(\boldsymbol{G}_{n}+\boldsymbol{\Delta}_{G} \boldsymbol{\Lambda}+\boldsymbol{G}_{n}(\boldsymbol{\Lambda}-\boldsymbol{I})\right) \boldsymbol{u},
\end{aligned}
$$




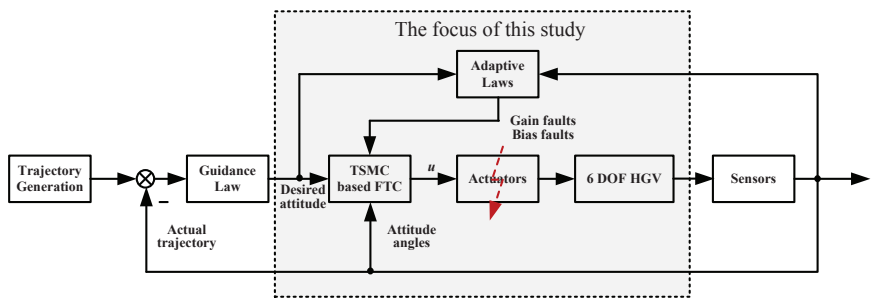

Fig. 1. The schematic illustration of the studied HGV FTC.

where $\boldsymbol{I}$ is a $3 \times 3$ identity matrix.

Assumption 1: It is assumed that the boundedness of $\dot{f}_{\mu}, \dot{f}_{\alpha}$, and $\dot{f}_{\beta}$ is associated with the norm of system states. It can be further assumed that:

$$
\left\{\begin{array}{l}
\left\|\dot{\boldsymbol{f}}_{1}+\boldsymbol{\Delta}_{F}+\left(\boldsymbol{G}_{n}+\boldsymbol{\Delta}_{G}\right) \boldsymbol{\rho}\right\| \leq \varepsilon_{1}+\varepsilon_{2}\|\boldsymbol{x}\|, \\
\left\|\left(\boldsymbol{\Delta}_{G} \boldsymbol{\Lambda}+\boldsymbol{G}_{n}(\boldsymbol{\Lambda}-\boldsymbol{I})\right) \boldsymbol{G}_{n}^{-1}\right\| \leq \varepsilon_{3}<1,
\end{array}\right.
$$

where $\varepsilon_{1}, \varepsilon_{2}$, and $\varepsilon_{3}$ are positive scalars.

Remark 2: $\boldsymbol{f}_{1}=\left[f_{\mu}, f_{\alpha}, f_{\beta}\right]^{T}$ can be seen as an impact term of trajectory on the HGV attitude. Since the attitude dynamics is much faster than the translation motion, the values of $f_{\mu}$, $f_{\alpha}$, and $f_{\beta}$ are usually small. As can be seen from (29), the lumped additive uncertainty term $\dot{\boldsymbol{f}}_{1}+\boldsymbol{\Delta}_{F}+\left(\boldsymbol{G}_{n}+\boldsymbol{\Delta}_{G}\right) \boldsymbol{\rho}$ is dependent on the system states. $\dot{f}_{\mu}$ is not only greatly dependent on $\gamma, \mu, \alpha, \beta$, and $V$, but also on $p, q$, and $r$. Essentially, the HGV angles as well as the HGV velocity are bounded in typical HGV flight envelopes. Therefore, the bound of $\dot{f}_{\mu}$ is closely related to the norm of HGV states if $\beta \approx 0$, $\gamma \neq \pm 90^{\circ}$, and $V \neq 0$. The similar assumption can be applied to the boundedness of $\dot{f}_{\alpha}$ and $\dot{f}_{\beta}$.

Remark 3: The second inequality in (29) is essential such that the control signal $\boldsymbol{G}_{n} \boldsymbol{u}$ dominates the uncertain vector function $\left(\boldsymbol{\Delta}_{G} \boldsymbol{\Lambda}+\boldsymbol{G}_{n}(\boldsymbol{\Lambda}-\boldsymbol{I})\right) \boldsymbol{u}$, which is induced by the actuator anomalies and the control input matrix uncertainty. This condition in turn ensures that the actuators configured are capable of addressing the HGV uncertainty and fault issues. In addition, $\boldsymbol{G}_{n}$ and $\boldsymbol{\Delta}_{G}$ solely rely on HGV angles including $\alpha$ and $\beta$ which are bounded in typical flight envelopes, instead of the angular velocities $p, q$, and $r$. Hence, it is assumed that the second term of (29) is bounded.

Remark 4: Time-scale separation of the independent innerloop and outer-loop designs, stemming from Eq. (22), is typically enforced in most of the design approaches. It is difficult to guarantee the finite-time stability of the overall closed-loop system. By contrast, Eq. (28) is integrated by Eqs. (13) and (20), which provides the basis of the proposed composite-loop design. The proof procedure of the finite-time stability by virtue of multivariable TSMC and homogeneity theory is presented in Section IV.

\section{Fault-Tolerant Control Design of a HGV}

The system depicted in Fig. 1 is composed by the guidance and control units. Generally, the guidance system generates the commands of the bank angle, AOA, and sideslip angle. The FTC scheme that is the main focus of this study outputs the actuator commands necessary to track the desired attitude and to handle actuator malfunctions. Two problems are addressed in the following sections. The first is the compositeloop design of FTC problem: construct the HGV FTC law against actuator malfunctions and model uncertainties, using the multivariable integral TSMC technique. The second is the problem of selecting the control parameters within the developed FTC scheme: determine the control parameters by exploring the adaptive tuning method.

\section{A. Multivariable TSMC based FTC Design}

Define the tracking error vector as:

$$
\boldsymbol{\sigma}=\left[\begin{array}{c}
\sigma_{\mu} \\
\sigma_{\alpha} \\
\sigma_{\beta}
\end{array}\right]=\left[\begin{array}{l}
\mu-\mu_{d} \\
\alpha-\alpha_{d} \\
\beta-\beta_{d}
\end{array}\right] .
$$

The multivariable integral terminal sliding mode manifold is defined as:

$$
\boldsymbol{S}=\left[\begin{array}{c}
S_{\mu} \\
S_{\alpha} \\
S_{\beta}
\end{array}\right]=\dot{\boldsymbol{\sigma}}+\int_{0}^{t} k_{1}\|\boldsymbol{\sigma}\|^{r_{1}} \frac{\boldsymbol{\sigma}}{\|\boldsymbol{\sigma}\|}+k_{2}\|\dot{\boldsymbol{\sigma}}\|^{r_{2}} \frac{\dot{\boldsymbol{\sigma}}}{\|\dot{\boldsymbol{\sigma}}\|} d \tau,
$$

where $r_{2} \in(0,1), r_{1}=2 r_{2} /\left(2-r_{2}\right)$, and $k_{1}, k_{2}>0$. The multivariable integral TSMC based FTC aims at steering the tracking error vector $\boldsymbol{\sigma}$ to the origin along $\boldsymbol{S}=\mathbf{0}$ in finite time, under actuator faults and model uncertainties.

Theorem 1: The HGV FTC law is formed as:

$$
\boldsymbol{u}=\boldsymbol{u}_{b}+\boldsymbol{u}_{d}
$$

where

$$
\begin{gathered}
\boldsymbol{u}_{b}=-\boldsymbol{G}_{n}^{-1}\left[\boldsymbol{F}_{n}-\boldsymbol{x}_{1, d}^{(2)}+k_{1}\|\boldsymbol{\sigma}\|^{r_{1}} \frac{\boldsymbol{\sigma}}{\|\boldsymbol{\sigma}\|}+k_{2}\|\dot{\boldsymbol{\sigma}}\|^{r_{2}} \frac{\dot{\boldsymbol{\sigma}}}{\|\dot{\boldsymbol{\sigma}}\|}\right], \\
\boldsymbol{u}_{d}=\left\{\begin{array}{cc}
-\boldsymbol{G}_{n}^{-1}\left(c_{1}+c_{2}\|\boldsymbol{x}\|+c_{3}\left\|\boldsymbol{F}_{n}-\boldsymbol{x}_{1, d}^{(2)}+k_{1}\right\| \boldsymbol{\sigma} \|^{r_{1}} \frac{\boldsymbol{\sigma}}{\|\boldsymbol{\sigma}\|}\right. \\
\left.+k_{2}\|\dot{\boldsymbol{\sigma}}\|^{r_{2}} \frac{\dot{\boldsymbol{\sigma}}}{\|\dot{\boldsymbol{\sigma}}\|} \|+\eta\right) \frac{\boldsymbol{S}}{\|\boldsymbol{S}\|} & \boldsymbol{S} \neq \mathbf{0} \\
\mathbf{0} & \boldsymbol{S}=\mathbf{0}
\end{array}\right.
\end{gathered}
$$

In Eqs. (32)-(34), $\boldsymbol{x}_{1, d}^{(2)}$ denotes the second time derivative of the desired $\boldsymbol{x}_{1}$ (i.e. $\boldsymbol{x}_{1, d}=\left[\mu_{d}, \alpha_{d}, \beta_{d}\right]^{T}$ ). $c_{1}, c_{2}, c_{3}$, and $\eta$ are the design parameters which are chosen as:

$$
c_{1}=\frac{\varepsilon_{1}}{1-\varepsilon_{3}}, c_{2}=\frac{\varepsilon_{2}}{1-\varepsilon_{3}}, c_{3}=\frac{\varepsilon_{3}}{1-\varepsilon_{3}}, \eta>0 .
$$

Therefore, the designed FTC law ensures that the tracking error vector $\boldsymbol{\sigma}$ is driven to the origin along $\boldsymbol{S}=\mathbf{0}$ in finite time regardless of actuator faults and model uncertainties.

Proof. Consider the following Lyapunov function:

$$
V_{1}=\frac{\boldsymbol{S}^{T} \boldsymbol{S}}{2} .
$$

The time derivative of $V_{1}$ for $\boldsymbol{S} \neq \mathbf{0}$ is:

$$
\begin{aligned}
\dot{V}_{1}= & \boldsymbol{S}^{T}\left(\ddot{\boldsymbol{\sigma}}+k_{1}\|\boldsymbol{\sigma}\|^{r_{1}} \frac{\boldsymbol{\sigma}}{\|\boldsymbol{\sigma}\|}+k_{2}\|\dot{\boldsymbol{\sigma}}\|^{r_{2}} \frac{\dot{\boldsymbol{\sigma}}}{\|\dot{\boldsymbol{\sigma}}\|}\right) \\
= & \boldsymbol{S}^{T}\left(\boldsymbol{F}_{n}+\dot{\boldsymbol{f}}_{1}+\boldsymbol{\Delta}_{F}+\left(\boldsymbol{G}_{n}+\boldsymbol{\Delta}_{G}\right) \boldsymbol{\rho}\right. \\
& +\left(\boldsymbol{G}_{n}+\boldsymbol{\Delta}_{G} \boldsymbol{\Lambda}+\boldsymbol{G}_{n}(\boldsymbol{\Lambda}-\boldsymbol{I})\right) \boldsymbol{u}-\boldsymbol{x}_{1, d}^{(2)} \\
& \left.+k_{1}\|\boldsymbol{\sigma}\|^{r_{1}} \frac{\boldsymbol{\sigma}}{\|\boldsymbol{\sigma}\|}+k_{2}\|\dot{\boldsymbol{\sigma}}\|^{r_{2}} \frac{\dot{\boldsymbol{\sigma}}}{\|\boldsymbol{\dot { \boldsymbol { \sigma } }}\|}\right) .
\end{aligned}
$$


Substituting (33)-(34) into (37) yields:

$$
\begin{aligned}
& \dot{V}_{1}=\boldsymbol{S}^{T}\left(\boldsymbol{F}_{n}+\dot{\boldsymbol{f}}_{1}+\boldsymbol{\Delta}_{F}+\left(\boldsymbol{G}_{n}+\boldsymbol{\Delta}_{G}\right) \boldsymbol{\rho}\right. \\
& -\left(\boldsymbol{G}_{n}+\boldsymbol{\Delta}_{G} \boldsymbol{\Lambda}+\boldsymbol{G}_{n}(\boldsymbol{\Lambda}-\boldsymbol{I})\right) \boldsymbol{G}_{n}^{-1}\left(\boldsymbol{F}_{n}-\boldsymbol{x}_{1, d}^{(2)}\right. \\
& +k_{1}\|\boldsymbol{\sigma}\|^{r_{1}} \frac{\boldsymbol{\sigma}}{\|\boldsymbol{\sigma}\|}+k_{2}\|\dot{\boldsymbol{\sigma}}\|^{r_{2}} \frac{\dot{\boldsymbol{\sigma}}}{\|\dot{\boldsymbol{\sigma}}\|}+\left(c_{1}+c_{2}\|\boldsymbol{x}\|\right. \\
& +c_{3}\left\|\boldsymbol{F}_{n}-\boldsymbol{x}_{1, d}^{(2)}+k_{1}\right\| \boldsymbol{\sigma}\left\|^{r_{1}} \frac{\boldsymbol{\sigma}}{\|\boldsymbol{\sigma}\|}+k_{2}\right\| \dot{\boldsymbol{\sigma}}\left\|^{r_{2}} \frac{\dot{\boldsymbol{\sigma}}}{\|\dot{\boldsymbol{\sigma}}\|}\right\| \\
& \left.\left.+\eta) \frac{\boldsymbol{S}}{\|\boldsymbol{S}\|}\right)+k_{1}\|\boldsymbol{\sigma}\|^{r_{1}} \frac{\boldsymbol{\sigma}}{\|\boldsymbol{\sigma}\|}+k_{2}\|\dot{\boldsymbol{\sigma}}\|^{r_{2}} \frac{\dot{\boldsymbol{\sigma}}}{\|\dot{\boldsymbol{\sigma}}\|}-\boldsymbol{x}_{1, d}^{(2)}\right) \\
& =\boldsymbol{S}^{T}\left(\dot{\boldsymbol{f}}_{1}+\boldsymbol{\Delta}_{F}+\left(\boldsymbol{G}_{n}+\boldsymbol{\Delta}_{G}\right) \boldsymbol{\rho}-\left(c_{1}+c_{2}\|\boldsymbol{x}\|\right.\right. \\
& +c_{3}\left\|\boldsymbol{F}_{n}-\boldsymbol{x}_{1, d}^{(2)}+k_{1}\right\| \boldsymbol{\sigma}\left\|^{r_{1}} \frac{\boldsymbol{\sigma}}{\|\boldsymbol{\sigma}\|}+k_{2}\right\| \dot{\boldsymbol{\sigma}}\left\|^{r_{2}} \frac{\dot{\boldsymbol{\sigma}}}{\|\dot{\boldsymbol{\sigma}}\|}\right\| \\
& +\eta) \frac{\boldsymbol{S}}{\|\boldsymbol{S}\|}-\left(\boldsymbol{\Delta}_{G} \boldsymbol{\Lambda}+\boldsymbol{G}_{n}(\boldsymbol{\Lambda}-\boldsymbol{I})\right) \boldsymbol{G}_{n}^{-1}\left(\boldsymbol{F}_{n}-\boldsymbol{x}_{1, d}^{(2)}\right. \\
& +k_{1}\|\boldsymbol{\sigma}\|^{r_{1}} \frac{\boldsymbol{\sigma}}{\|\boldsymbol{\sigma}\|}+k_{2}\|\dot{\boldsymbol{\sigma}}\|^{r_{2}} \frac{\dot{\boldsymbol{\sigma}}}{\|\dot{\boldsymbol{\sigma}}\|}+\left(c_{1}+c_{2}\|\boldsymbol{x}\|\right. \\
& +c_{3}\left\|\boldsymbol{F}_{n}-\boldsymbol{x}_{1, d}^{(2)}+k_{1}\right\| \boldsymbol{\sigma}\left\|^{r_{1}} \frac{\boldsymbol{\sigma}}{\|\boldsymbol{\sigma}\|}+k_{2}\right\| \dot{\boldsymbol{\sigma}}\left\|^{r_{2}} \frac{\dot{\boldsymbol{\sigma}}}{\|\dot{\boldsymbol{\sigma}}\|}\right\| \\
& \left.\left.+\eta) \frac{\boldsymbol{S}}{\|\boldsymbol{S}\|}\right)\right) \text {. }
\end{aligned}
$$

Using the condition (29) and Eq. (38) can achieve that:

$$
\begin{aligned}
\dot{V}_{1} & \leq\|\boldsymbol{S}\|\left(\varepsilon_{1}+\varepsilon_{2}\|\boldsymbol{x}\|\right)-\|\boldsymbol{S}\|\left(c_{1}+c_{2}\|\boldsymbol{x}\|+c_{3}\right. \\
& \left.\cdot\left\|\boldsymbol{F}_{n}-\boldsymbol{x}_{1, d}^{(2)}+k_{1}\right\| \boldsymbol{\sigma}\left\|^{r_{1}} \frac{\boldsymbol{\sigma}}{\|\boldsymbol{\sigma}\|}+k_{2}\right\| \dot{\boldsymbol{\sigma}}\left\|^{r_{2}} \frac{\dot{\boldsymbol{\sigma}}}{\|\dot{\boldsymbol{\sigma}}\|}\right\|+\eta\right) \\
& +\varepsilon_{3}\|\boldsymbol{S}\|\left\|\boldsymbol{F}_{n}-\boldsymbol{x}_{1, d}^{(2)}+k_{1}\right\| \boldsymbol{\sigma}\left\|^{r_{1}} \frac{\boldsymbol{\sigma}}{\|\boldsymbol{\sigma}\|}+k_{2}\right\| \dot{\boldsymbol{\sigma}}\left\|^{r_{2}} \frac{\dot{\boldsymbol{\sigma}}}{\|\dot{\boldsymbol{\sigma}}\|}\right\| \\
& +\varepsilon_{3}\|\boldsymbol{S}\|\left(c_{1}+c_{2}\|\boldsymbol{x}\|\right. \\
& \left.+c_{3}\left\|\boldsymbol{F}_{n}-\boldsymbol{x}_{1, d}^{(2)}+k_{1}\right\| \boldsymbol{\sigma}\left\|^{r_{1}} \frac{\boldsymbol{\sigma}}{\|\boldsymbol{\sigma}\|}+k_{2}\right\| \dot{\boldsymbol{\sigma}}\left\|^{r_{2}} \frac{\dot{\boldsymbol{\sigma}}}{\|\dot{\boldsymbol{\sigma}}\|}\right\|+\eta\right) \\
& =\|\boldsymbol{S}\|\left(\varepsilon_{1}-c_{1}\left(1-\varepsilon_{3}\right)-\left(1-\varepsilon_{3}\right) \eta\right) \\
& +\|\boldsymbol{S}\|\|\boldsymbol{x}\|\left(\varepsilon_{2}-c_{2}\left(1-\varepsilon_{3}\right)\right) \\
& +\|\boldsymbol{S}\|\left\|\boldsymbol{F}_{n}-\boldsymbol{x}_{1, d}^{(2)}+k_{1}\right\| \boldsymbol{\sigma}\left\|^{r_{1}} \frac{\boldsymbol{\sigma}}{\|\boldsymbol{\sigma}\|}+k_{2}\right\| \dot{\boldsymbol{\sigma}}\left\|^{r_{2}} \frac{\dot{\boldsymbol{\sigma}}}{\|\dot{\boldsymbol{\sigma}}\|}\right\| \\
& \cdot\left(\varepsilon_{3}-\left(1-\varepsilon_{3}\right) c_{3}\right) \\
& =-\left(1-\varepsilon_{3}\right) \eta\|\boldsymbol{S}\| \\
& =-\left(1-\varepsilon_{3}\right) \eta \sqrt{2} V_{1}^{\frac{1}{2}} .
\end{aligned}
$$

As a result, $V_{1}=0$ when $\boldsymbol{S}=\mathbf{0}$. It is concluded that the tracking error $\boldsymbol{\sigma}$ can reach the sliding manifold $\boldsymbol{S}=\mathbf{0}$ in finite time and remain there in spite of the actuator anomalies and model uncertainties. On the sliding manifold, the equivalent dynamics can be obtained by writing $\dot{\boldsymbol{S}}=\mathbf{0}$ as follows:

$$
\ddot{\boldsymbol{\sigma}}+k_{1}\|\boldsymbol{\sigma}\|^{r_{1}} \frac{\boldsymbol{\sigma}}{\|\boldsymbol{\sigma}\|}+k_{2}\|\dot{\boldsymbol{\sigma}}\|^{r_{2}} \frac{\dot{\boldsymbol{\sigma}}}{\|\dot{\boldsymbol{\sigma}}\|}=\mathbf{0} \text {. }
$$

In the sequel, it is proved that the dynamics of (40) is finitetime stable. By letting $\zeta_{1}=\sigma$ and $\zeta_{2}=\dot{\sigma}$, the sliding dynamics can be represented as:

$$
\left\{\begin{array}{l}
\dot{\zeta}_{1}=\boldsymbol{\zeta}_{2}, \\
\dot{\zeta}_{2}=-k_{1} \frac{\zeta_{1}}{\left\|\boldsymbol{\zeta}_{1}\right\|^{1-r_{1}}}-k_{2} \frac{\zeta_{2}}{\left\|\boldsymbol{\zeta}_{2}\right\|^{1-r_{2}}} .
\end{array}\right.
$$

Consider a Lyapunov function as:

$$
V_{2}=k_{1} \frac{\left\|\boldsymbol{\zeta}_{1}\right\|^{r_{1}+1}}{r_{1}+1}+\frac{\left\|\boldsymbol{\zeta}_{2}\right\|^{2}}{2}
$$

The time derivative of $V_{2}$ along (41) is:

$$
\begin{aligned}
\dot{V}_{2}= & k_{1}\left\|\boldsymbol{\zeta}_{1}\right\|^{r_{1}-1} \dot{\boldsymbol{\zeta}}_{1}^{T} \boldsymbol{\zeta}_{1}-\boldsymbol{\zeta}_{2}^{T}\left(k_{1} \frac{\boldsymbol{\zeta}_{1}}{\left\|\boldsymbol{\zeta}_{1}\right\|^{1-r_{1}}}+k_{2} \frac{\boldsymbol{\zeta}_{2}}{\left\|\boldsymbol{\zeta}_{2}\right\|^{1-r_{2}}}\right) \\
= & k_{1}\left\|\boldsymbol{\zeta}_{1}\right\|^{r_{1}-1} \dot{\boldsymbol{\zeta}}_{1}^{T} \boldsymbol{\zeta}_{1}-k_{1}\left\|\boldsymbol{\zeta}_{1}\right\|^{r_{1}-1} \boldsymbol{\zeta}_{2}^{T} \boldsymbol{\zeta}_{1} \\
& -k_{2}\left\|\boldsymbol{\zeta}_{2}\right\|^{r_{2}-1} \boldsymbol{\zeta}_{2}^{T} \boldsymbol{\zeta}_{2} \\
= & -k_{2}\left\|\boldsymbol{\zeta}_{2}\right\|^{r_{2}+1}
\end{aligned}
$$

Applying LaSalle's invariance principle, the set $\left\{\left(\boldsymbol{\zeta}_{1}, \boldsymbol{\zeta}_{2}\right)\right.$ : $\left.\dot{V}_{2}\left(\boldsymbol{\zeta}_{1}, \boldsymbol{\zeta}_{2}\right)=0\right\}$ consists of $\boldsymbol{\zeta}_{2}=\mathbf{0}$, and the only invariant set inside $\zeta_{2}=\mathbf{0}$ is the origin $\boldsymbol{\zeta}_{1}=\boldsymbol{\zeta}_{2}=\mathbf{0}$. Thus, the asymptotic convergence of $\zeta_{1}$ and $\zeta_{2}$ to zero is guaranteed. Further, considering the vector field (41) and the dilation $\left(1,1,1, \frac{1}{2-r_{2}}, \frac{1}{2-r_{2}}, \frac{1}{2-r_{2}}\right)$, one can conclude that the vector field (41) is homogeneous of degree $\frac{r_{2}-1}{2-r_{2}}<0$. According to Lemma 1, it can be concluded that system (40) is globally finite-time stable. Thus, the tracking error $\sigma$ can be driven to the origin along $\boldsymbol{S}=\mathbf{0}$ in finite time, although actuator faults take place. This completes the proof.

Remark 5: In the sliding manifold definition (31) and the proposed FTC (32)-(34), the derivatives of $\dot{\sigma}$ can be estimated on-line by the robust exact finite-time convergent differentiator [30]. The differentiator can be implemented if the higher order derivatives of the input are bounded and the finitetime escape does not exist. The differentiator transient can be driven short enough by appropriately tuning the differentiator parameters. As argued in $[30,31]$, the differentiator can satisfy most of feedback requirement, if the convergence of the used differentiator is adequately fast and accurate.

Remark 6: The uncertainty term, $\boldsymbol{\Delta}_{G}$, cannot be ignored since the limits of wind tunnel and flight tests determine a partial knowledge of $\mathrm{HGV}$ aerodynamic derivatives. In addition, actuator faults may be caused by the reentry thermal environment and ablation. This study with explicit consideration of both difficulties can be seen a further step of the existing literature.

Remark 7: Time-scale separation of the independent innerloop and outer-loop designs, stemming from Eq. (22), is typically enforced in most of the design approaches [25, 26]. One needs to develop two controllers corresponding to the separated loops. However, it is difficult to guarantee the finitetime stability of the overall closed-loop system. In this study, Eq. (28) is integrated by Eqs. (13) and (20), which provides the basis of the proposed composite-loop design.

Remark 8: In [23, 32], multivariable TSMC design is discussed for hypersonic vehicles. The sliding manifold [23, 32] is essentially established by a decoupled treatment. Instead, based on sliding manifold of vector expression, the approach developed in this paper has twofold benefits: 1) the problem related to the decoupled design is avoided; and 2) the multivariable FTC can maintain the globally finite-time stability under actuator malfunctions. These improvements have the potential to enhance the safety of operational HGVs.

Remark 9: The second term of the right hand side of Eq. (40) can be seen as a proportional-like control term, while the third one of the right hand side of Eq. (40) is considered as a differential-like control term. Eq. (40) can guarantee both $\boldsymbol{\sigma}$ and $\dot{\boldsymbol{\sigma}}$ converge to zero in finite time, as detailed in the proof procedure of Theorem 1. The advantages of such kind of sliding manifold are: 1) a more concise solution than a decoupled collection of single variable structures is achieved, facilitating multivariable FTC design; 2) similar to the conventional terminal sliding manifold, the finite-time convergence to zero can be ensured in the sliding mode; and 3 ) as shown in the sliding manifold (31), the fractional power integral terms $\|\boldsymbol{\sigma}\|^{r_{1}} \frac{\boldsymbol{\sigma}}{\|\boldsymbol{\sigma}\|}$ and $\|\dot{\boldsymbol{\sigma}}\|^{r_{2}} \frac{\dot{\boldsymbol{\sigma}}}{\|\dot{\boldsymbol{\sigma}}\|}$ are "hidden" behind the integral action. There are no negative fractional power terms appearing in the FTC law (32)-(34). In other words, the singularity problem in TSMC can be avoided completely by adopting Eq. (31). In this sense, the proposed approach is more straightforward as compared to those in [33-35]. 


\section{B. Adaptive Multivariable TSMC based FTC Design}

The FTC strategy against HGV actuator faults is developed, as indicated in Eqs. (32)-(34) of Theorem 1. However, the control parameters $c_{1}, c_{2}$, and $c_{3}$ may not be obtained due to the complexity of the uncertainties and actuators faults. To better address the selection of $c_{1}, c_{2}$, and $c_{3}$, an adaptation algorithm within the FTC scheme is proposed in Theorem 2.

Theorem 2: Given the faulty HGV model in Eq. (27) and a FTC law constructed by Eqs. (32)-(34), the control parameters $c_{1}, c_{2}$, and $c_{3}$ can be estimated by:

$$
\begin{gathered}
\dot{\hat{c}}_{1}=\left\{\begin{array}{cc}
\frac{1}{\gamma_{1}}, & \boldsymbol{S} \neq \mathbf{0}, \\
0, & \boldsymbol{S}=\mathbf{0},
\end{array}\right. \\
\dot{\hat{c}}_{2}=\left\{\begin{array}{cc}
\frac{1}{\gamma_{2}}\|\boldsymbol{x}\|, & \boldsymbol{S} \neq \mathbf{0}, \\
0, & \boldsymbol{S}=\mathbf{0},
\end{array}\right. \\
\dot{\hat{c}}_{3}= \begin{cases}\frac{1}{\gamma_{3}}\left\|\boldsymbol{F}_{n}-\boldsymbol{x}_{1, d}^{(2)}+k_{1}\right\| \boldsymbol{\sigma}\left\|^{r_{1}} \frac{\boldsymbol{\sigma}}{\|\boldsymbol{\sigma}\|}+k_{2}\right\| \dot{\boldsymbol{\sigma}} \|^{r_{2}} \frac{\boldsymbol{\sigma}}{\|\boldsymbol{\sigma}\| \|} \\
\boldsymbol{S} \neq \mathbf{0}, \\
0, & \boldsymbol{S}=\mathbf{0} .\end{cases}
\end{gathered}
$$

Note that $\gamma_{i}$ are positive design parameters, $\hat{c}_{i}(0)>0$, and $i=1,2,3$.

Proof. The parameter errors are defined as:

$$
\tilde{c}_{1}=\hat{c}_{1}-\frac{\varepsilon_{1}}{1-\varepsilon_{3}}, \tilde{c}_{2}=\hat{c}_{2}-\frac{\varepsilon_{2}}{1-\varepsilon_{3}}, \tilde{c}_{3}=\hat{c}_{3}-\frac{\varepsilon_{3}}{1-\varepsilon_{3}} .
$$

A Lyapunov function is selected as:

$$
V_{3}=\|\boldsymbol{S}\|+\frac{1-\varepsilon_{2}}{2} \gamma_{1} \tilde{c}_{1}^{2}+\frac{1-\varepsilon_{2}}{2} \gamma_{2} \tilde{c}_{2}^{2}+\frac{1-\varepsilon_{2}}{2} \gamma_{3} \tilde{c}_{3}^{2} .
$$

Noting that $\dot{\tilde{c}}_{i}=\dot{\hat{c}}_{i}, i=1,2,3$, one can obtain the time derivative of $V_{3}$ along the trajectories of (27) when $\boldsymbol{S} \neq \mathbf{0}$

$$
\begin{aligned}
\dot{V}_{3}= & \frac{\boldsymbol{S}^{T} \dot{\boldsymbol{S}}}{\|\boldsymbol{S}\|}+\left(1-\varepsilon_{3}\right) \gamma_{1} \tilde{c}_{1} \dot{\hat{c}}_{1}+\left(1-\varepsilon_{3}\right) \gamma_{2} \tilde{c}_{2} \dot{\hat{c}}_{2} \\
& +\left(1-\varepsilon_{3}\right) \gamma_{3} \tilde{c}_{3} \hat{\hat{c}}_{3} \\
= & \frac{\boldsymbol{S}^{T}}{\|\boldsymbol{S}\|}\left(\dot{\boldsymbol{f}}_{1}+\boldsymbol{\Delta}_{F}+\left(\boldsymbol{G}_{n}+\boldsymbol{\Delta}_{G}\right) \boldsymbol{\rho}-\left(\hat{c}_{1}+\hat{c}_{2}\|\boldsymbol{x}\|\right.\right. \\
& +\hat{c}_{3}\left\|\boldsymbol{F}_{n}-\boldsymbol{x}_{1, d}^{(2)}+k_{1}\right\| \boldsymbol{\sigma} \|^{r_{1}} \frac{\boldsymbol{\sigma}}{\|\boldsymbol{\sigma}\|} \\
& \left.+k_{2}\|\boldsymbol{\boldsymbol { \sigma }}\|^{r_{2}} \frac{\dot{\boldsymbol{\sigma}}}{\|\dot{\boldsymbol{\sigma}}\|} \| \eta\right) \frac{\boldsymbol{S}}{\|\boldsymbol{S}\|}-\left(\boldsymbol{\Delta} \boldsymbol{\Delta}_{G} \boldsymbol{\Lambda}+\boldsymbol{G}_{n}(\boldsymbol{\Lambda}-\boldsymbol{I})\right) \\
& +\boldsymbol{G}_{n}^{-1}\left[\boldsymbol{F}_{n}-\boldsymbol{x}_{1, d}^{(2)}+k_{1}\|\boldsymbol{\sigma}\|^{r_{1}} \frac{\boldsymbol{\sigma}}{\|\boldsymbol{\sigma}\|}+k_{2}\|\boldsymbol{\boldsymbol { \sigma }}\|^{r_{2}} \frac{\dot{\boldsymbol{\sigma}}}{\|\dot{\boldsymbol{\sigma}}\|}\right] \\
& -\left(\boldsymbol{\Delta}_{G} \boldsymbol{\Lambda}+\boldsymbol{G}_{n}(\boldsymbol{\Lambda}-\boldsymbol{I})\right) \boldsymbol{G}_{n}^{-1}\left(\hat{c}_{1}+\hat{c}_{2}\|\boldsymbol{x}\|\right. \\
& +\hat{c}_{3}\left\|\boldsymbol{F}_{n}-\boldsymbol{x}_{1, d}^{(2)}+k_{1}\right\| \boldsymbol{\sigma}\left\|^{r_{1}} \frac{\boldsymbol{\sigma}}{\|\boldsymbol{\sigma}\|}+k_{2}\right\| \dot{\boldsymbol{\sigma}}\left\|^{r_{2}} \frac{\boldsymbol{\sigma}}{\|\dot{\boldsymbol{\sigma}}\|}\right\| \\
& \left.+\eta) \frac{\boldsymbol{S}}{\left\|\boldsymbol{S}_{\|}\right\|}\right)+\left(1-\varepsilon_{3}\right) \hat{c}_{1}-\varepsilon_{1}+\left(1-\varepsilon_{3}\right) \hat{c}_{2}\|\boldsymbol{x}\| \\
& -\varepsilon_{2}\|\boldsymbol{x}\|+\left(1-\varepsilon_{3}\right) \hat{c}_{3} \\
& \cdot\left\|\boldsymbol{F}_{n}-\boldsymbol{x}_{1, d}^{(2)}+k_{1}\right\| \boldsymbol{\sigma}\left\|^{r_{1}} \frac{\boldsymbol{\sigma}}{\|\boldsymbol{\sigma}\|}+k_{2}\right\| \dot{\boldsymbol{\sigma}}\left\|^{r_{2}} \frac{\dot{\boldsymbol{\sigma}}}{\|\dot{\boldsymbol{\sigma}}\|}\right\| \\
& -\varepsilon_{3}\left\|\boldsymbol{F}_{n}-\boldsymbol{x}_{1, d}^{(2)}+k_{1}\right\| \boldsymbol{\sigma}\left\|^{r_{1}} \frac{\boldsymbol{\sigma}}{\|\boldsymbol{\sigma}\|}+k_{2}\right\| \dot{\boldsymbol{\sigma}}\left\|^{r_{2}} \frac{\boldsymbol{\sigma}}{\|\dot{\boldsymbol{\sigma}}\|}\right\| .
\end{aligned}
$$

Applying the condition (29) to Eq. (49) gives:

$$
\begin{aligned}
& \dot{V}_{3} \leq\left\|\dot{\boldsymbol{f}}_{1}+\boldsymbol{\Delta}_{F}+\left(\boldsymbol{G}_{n}+\boldsymbol{\Delta}_{G}\right) \boldsymbol{\rho}\right\| \\
& +\left\|\left(\boldsymbol{\Delta}_{G} \boldsymbol{\Lambda}+\boldsymbol{G}_{n}(\boldsymbol{\Lambda}-\boldsymbol{I})\right) \boldsymbol{G}_{n}^{-1}\right\| \\
& \cdot\left\|\boldsymbol{F}_{n}-\boldsymbol{x}_{1, d}^{(2)}+k_{1}\right\| \boldsymbol{\sigma}\left\|^{r_{1}} \frac{\boldsymbol{\sigma}}{\|\boldsymbol{\sigma}\|}+k_{2}\right\| \dot{\boldsymbol{\sigma}}\left\|^{r_{1}} \frac{\dot{\boldsymbol{\sigma}}}{\|\dot{\boldsymbol{\sigma}}\|}\right\| \\
& -\left(\hat{c}_{1}+\hat{c}_{2}\|\boldsymbol{x}\|+\hat{c}_{3}\left\|\boldsymbol{F}_{n}-\boldsymbol{x}_{1, d}^{(2)}+k_{1}\right\| \boldsymbol{\sigma} \|^{r_{1}} \frac{\boldsymbol{\sigma}}{\|\boldsymbol{\sigma}\|}\right. \\
& \left.+k_{2}\|\dot{\boldsymbol{\sigma}}\|^{r_{1}} \frac{\dot{\boldsymbol{\sigma}}}{\|\dot{\boldsymbol{\sigma}}\|} \|+\eta\right)+\left\|\left(\boldsymbol{\Delta}_{G} \boldsymbol{\Lambda}+\boldsymbol{G}_{n}(\boldsymbol{\Lambda}-\boldsymbol{I})\right) \boldsymbol{G}_{n}^{-1}\right\| \\
& \cdot\left(\hat{c}_{1}+\hat{c}_{2}\|\boldsymbol{x}\|+\hat{c}_{3}\left\|\boldsymbol{F}_{n}-\boldsymbol{x}_{1, d}^{(2)}+k_{1}\right\| \boldsymbol{\sigma} \|^{r_{1}} \frac{\boldsymbol{\sigma}}{\|\boldsymbol{\sigma}\|}\right. \\
& \left.+k_{2}\|\dot{\boldsymbol{\sigma}}\|^{r_{1}} \frac{\dot{\boldsymbol{\sigma}}}{\|\dot{\boldsymbol{\sigma}}\|} \|+\eta\right)+\left(1-\varepsilon_{3}\right) \hat{c}_{1}-\varepsilon_{1} \\
& +\left(1-\varepsilon_{3}\right) \hat{c}_{2}\|\boldsymbol{x}\|-\varepsilon_{2}\|\boldsymbol{x}\|+\left(1-\varepsilon_{3}\right) \hat{c}_{3} \\
& \cdot\left\|\boldsymbol{F}_{n}-\boldsymbol{x}_{1, d}^{(2)}+k_{1}\right\| \boldsymbol{\sigma}\left\|^{r_{1}} \frac{\boldsymbol{\sigma}}{\|\boldsymbol{\sigma}\|}+k_{2}\right\| \dot{\boldsymbol{\sigma}}\left\|^{r_{2}} \frac{\dot{\boldsymbol{\sigma}}}{\|\dot{\boldsymbol{\sigma}}\|}\right\| \\
& -\varepsilon_{3}\left\|\boldsymbol{F}_{n}-\boldsymbol{x}_{1, d}^{(2)}+k_{1}\right\| \boldsymbol{\sigma}\left\|^{r_{1}} \frac{\boldsymbol{\sigma}}{\|\boldsymbol{\sigma}\|}+k_{2}\right\| \dot{\boldsymbol{\sigma}}\left\|^{r_{2}} \frac{\dot{\boldsymbol{\sigma}}}{\|\dot{\boldsymbol{\sigma}}\|}\right\| \\
& \leq \varepsilon_{1}+\varepsilon_{2}\|\boldsymbol{x}\|-\left(\hat{c}_{1}+\hat{c}_{2}\|\boldsymbol{x}\|+\hat{c}_{3}\right. \\
& \text { - } \left.\left\|\boldsymbol{F}_{n}-\boldsymbol{x}_{1, d}^{(2)}+k_{1}\right\| \boldsymbol{\sigma}\left\|^{r_{1}} \frac{\boldsymbol{\sigma}}{\|\boldsymbol{\sigma}\|}+k_{2}\right\| \dot{\boldsymbol{\sigma}}\left\|^{r_{2}} \frac{\dot{\boldsymbol{\sigma}}}{\|\boldsymbol{\boldsymbol { \sigma }}\|}\right\|+\eta\right) \\
& +\varepsilon_{3}\left\|\boldsymbol{F}_{n}-\boldsymbol{x}_{1, d}^{(2)}+k_{1}\right\| \boldsymbol{\sigma}\left\|^{r_{1}} \frac{\boldsymbol{\sigma}}{\|\boldsymbol{\sigma}\|}+k_{2}\right\| \dot{\boldsymbol{\sigma}}\left\|^{r_{2}} \frac{\dot{\boldsymbol{\sigma}}}{\|\dot{\boldsymbol{\sigma}}\|}\right\| \\
& +\varepsilon_{3}\left(\hat{c}_{1}+\hat{c}_{2}\|\boldsymbol{x}\|+\hat{c}_{3}\left\|\boldsymbol{F}_{n}-\boldsymbol{x}_{1, d}^{(2)}+k_{1}\right\| \boldsymbol{\sigma} \|^{r_{1}} \frac{\boldsymbol{\sigma}}{\|\boldsymbol{\sigma}\|}\right. \\
& \left.+k_{2}\|\dot{\boldsymbol{\sigma}}\|^{r_{2}} \frac{\dot{\boldsymbol{\sigma}}}{\|\dot{\boldsymbol{\sigma}}\|} \|+\eta\right)+\left(1-\varepsilon_{3}\right) \hat{c}_{1}-\varepsilon_{1} \\
& +\left(1-\varepsilon_{3}\right) \hat{c}_{2}\|\boldsymbol{x}\|-\varepsilon_{2}\|\boldsymbol{x}\|+\left(1-\varepsilon_{3}\right) \hat{c}_{3} \\
& \text {. }\left\|\boldsymbol{F}_{n}-\boldsymbol{x}_{1, d}^{(2)}+k_{1}\right\| \boldsymbol{\sigma}\left\|^{r_{1}} \frac{\boldsymbol{\sigma}}{\|\boldsymbol{\sigma}\|}+k_{2}\right\| \dot{\boldsymbol{\sigma}}\left\|^{r_{2}} \frac{\dot{\boldsymbol{\sigma}}}{\|\dot{\boldsymbol{\sigma}}\|}\right\| \\
& -\varepsilon_{3}\left\|\boldsymbol{F}_{n}-\boldsymbol{x}_{1, d}^{(2)}+k_{1}\right\| \boldsymbol{\sigma}\left\|^{r_{1}} \frac{\boldsymbol{\sigma}}{\|\boldsymbol{\sigma}\|}+k_{2}\right\| \dot{\boldsymbol{\sigma}}\left\|^{r_{2}} \frac{\dot{\boldsymbol{\sigma}}}{\|\boldsymbol{\dot { \boldsymbol { \sigma } }}\|}\right\| \\
& =-\left(1-\varepsilon_{3}\right) \eta<0 \text {. }
\end{aligned}
$$

The condition (50) implies that the values of $V_{3}$ and $S$ will approach to zero in finite time $t_{f}$, i.e., $V_{3}\left(t_{f}\right)=0$, and it can be verified that $t_{f} \leq t_{0}+\frac{V_{3}\left(t_{0}\right)}{\eta\left(1-\varepsilon_{3}\right)}$. Since the value of $V_{3}$ is bounded, $\tilde{c}_{i}$ (and hence $\hat{c}_{i}$ ) are all bounded. Moreover, in accordance with Eq. (45), the solution of $\hat{c}_{2}$ is:

$$
\hat{c}_{2}(t)=\frac{1}{\gamma_{2}} \int_{t_{0}}^{t_{f}}\|\boldsymbol{x}(\tau)\| d \tau+\hat{c}_{2}(0), \quad \boldsymbol{S} \neq \mathbf{0} .
$$

When $\hat{c}_{2}$ is bounded and the integral is nonnegative, the state variable $\boldsymbol{x}$ of (27) is bounded for $t_{0}<t<t_{f}$.

Remark 10: $\boldsymbol{u}_{b}$ is considered as nominal control, while $\boldsymbol{u}_{d}$ is designed to compensate for the detrimental impact of HGV actuator faults and model uncertainties. The parameters $c_{1}, c_{2}$, and $c_{3}$ in Eq. (33) are linked to the HGV actuator faults. It is worth to emphasize that the values of $c_{1}, c_{2}$, and $c_{3}$ are tuned in response to the knowledge of the current status of the HGV.

Remark 11: It is noted that $\boldsymbol{u}_{d}$ contains a switching term $\frac{S}{\|S\|}$. Due to nonlinearities, noisy measurements, and nonideal switching, the control chattering exists. For alleviating the chattering phenomenon, one option is to replace the switching function $\frac{\boldsymbol{S}}{\|\boldsymbol{S}\|}$ of (34) as:

$$
\operatorname{sat}(\boldsymbol{S}, \Phi)=\left\{\begin{array}{cc}
\frac{S}{\Phi} & \|\boldsymbol{S}\| \leq \Phi \\
\frac{\boldsymbol{S}}{\|\boldsymbol{S}\|} & \|\boldsymbol{S}\|>\Phi
\end{array},\right.
$$

where $\Phi$ is a small positive constant. Here, $\Phi$ helps define a boundary layer about the sliding manifold $\boldsymbol{S}=\mathbf{0}$ inside which an acceptably close approximation to ideal sliding takes place. Provided the states evolve with time inside the boundary layer, no adaptation of the switching gains takes place. If a fault occurs that starts to make the sliding motion degrade so that the states evolve outside the boundary, i.e., $\|\boldsymbol{S}\|>\Phi$, then the gains $\hat{c}_{i}$ increase in magnitude to force the states back into 
the boundary layer around the sliding manifold. The adaptive tuning laws (44)-(46) are modified as [36, 37]:

$$
\begin{gathered}
\dot{\hat{c}}_{1}=\left\{\begin{array}{cl}
\frac{1}{\gamma_{1},} & \|\boldsymbol{S}\|>\Phi \\
0, & \|\boldsymbol{S}\| \leq \Phi
\end{array}\right. \\
\dot{\hat{c}}_{2}=\left\{\begin{array}{cl}
\frac{1}{\gamma_{2}}\|\boldsymbol{x}\|, & \|\boldsymbol{S}\|>\Phi \\
0, & \|\boldsymbol{S}\| \leq \Phi,
\end{array}\right. \\
\dot{\hat{c}}_{3}=\left\{\begin{array}{cl}
\frac{1}{\gamma_{3}}\left\|\boldsymbol{F}_{n}-\boldsymbol{x}_{1, d}^{(2)}+k_{1}\right\| \boldsymbol{\sigma}\left\|^{r_{1}} \frac{\boldsymbol{\sigma}}{\|\boldsymbol{\sigma}\|}+k_{2}\right\| \dot{\boldsymbol{\sigma}} \|^{r_{2}} \frac{\dot{\boldsymbol{\sigma}}}{\|\boldsymbol{\dot { \boldsymbol { \sigma } } \|}\|} \\
0, & \|\boldsymbol{S}\|>\Phi
\end{array}\right.
\end{gathered}
$$

The designer has to tradeoff between tracking accuracy and chattering phenomenon when adjusting the parameter $\Phi$. A time-varying dead-zone modification is proposed in [38]. The resulting method can improve the adaptive law robustness to measurement or system noises.

Remark 12: More recently, adaptive continuous HOSMC methods have been developed [9, 39-42], which provide a novel dual-layer adaptive mechanism. Instead of artificially increasing the relative degree and using the virtual control input, the adaptive continuous HOSMC can obtain continuous control signals directly, and the bounds of the uncertainties are not needed a priori. Meanwhile, novel design approaches for multivariable situations have been proposed in [43, 44].

\section{Simulation Results}

\section{A. HGV Flight Condition and Simulation Scenarios}

The initial flight conditions of the $\mathrm{HGV}$ are: $V(0)=$ $3000 \mathrm{~m} / \mathrm{s}, H=30000 \mathrm{~m}, \mu(0)=2^{\circ}, \alpha(0)=2^{\circ}, \beta(0)=2^{\circ}$, $p(0)=q(0)=r(0)=0$. The model uncertainties, actuator faults, and sensed signals with white noises are introduced to assess the performance of the developed FTC scheme.

1) The uncertainties corresponding to the roll, pitch, and yaw moments of inertia $\left(I_{x x}, I_{y y}, I_{z z}\right)$ are $10 \%$ of the nominal values. The maximal $20 \%$ mismatch exists in the HGV mass, $C_{l}, C_{m}$, and $C_{n}$, respectively.

2) Focusing on the fault pattern of the $\mathrm{HGV}$ actuators, the gain faults and bias faults are included with consideration of both time-invariant and time-varying cases, as can be found in Eqs. (56)-(57), respectively. Actuator time-invariant faults:

$$
\begin{aligned}
& \lambda_{1}=\left\{\begin{array}{cc}
1 & 0 \leq t<9 \\
0.75 & t>9
\end{array}, \rho_{1}=\left\{\begin{array}{cc}
0 & 0 \leq t<9 \\
2 & t>9
\end{array}\right.\right. \\
& \lambda_{2}=\left\{\begin{array}{cc}
1 & 0 \leq t<9 \\
0.75 & t>9
\end{array}, \rho_{2}=\left\{\begin{array}{cc}
0 & 0 \leq t<9 \\
-3 & t>9
\end{array}\right.\right. \\
& \lambda_{3}=\left\{\begin{array}{cc}
1 & 0 \leq t<9 \\
0.75 & t>9
\end{array}, \rho_{3}=\left\{\begin{array}{cc}
0 & 0 \leq t<9 \\
2 & t>9
\end{array}\right. \text {. }\right.
\end{aligned}
$$

Actuator time-varying faults:

$$
\begin{aligned}
& \lambda_{1}=\left\{\begin{array}{cc}
1 & 0 \leq t<9 \\
0.75-\frac{t-9}{60} & 9 \leq t \leq 15 \\
0.65 & t>15
\end{array},\right. \\
& \rho_{1}=\left\{\begin{array}{cc}
0 & 0 \leq t<9 \\
2+\frac{t-9}{3} & 9 \leq t \leq 15 \\
4 & t>15
\end{array},\right. \\
& \lambda_{2}=\left\{\begin{array}{cc}
1 & 0 \leq t<9 \\
0.75-\frac{t-9}{30} & 9 \leq t \leq 15 \\
0.55 & t>15
\end{array}\right. \\
& \rho_{2}=\left\{\begin{array}{cc}
0 & 0 \leq t<9 \\
-3-\frac{t-9}{6} & 9 \leq t \leq 15 \\
-4 & t>15
\end{array},\right. \\
& \lambda_{3}=\left\{\begin{array}{cc}
1 & 0 \leq t<9 \\
0.7-\frac{t-9}{30} & 9 \leq t \leq 15 \\
0.5 & t>15
\end{array},\right. \\
& \rho_{3}=\left\{\begin{array}{cc}
0 & 0 \leq t<9 \\
2+\frac{t-9}{3} & 9 \leq t \leq 15 \\
4 & t>15
\end{array} .\right.
\end{aligned}
$$

3) The white noise with a mean of 0 and covariance of 0.01 is injected into each measurement channel.

Two scenarios are studied to demonstrate the use of the algorithms for HGV attitude tracking control. 1) Scenario I: the model uncertainty, time-invariant actuator gain and bias faults, and measurement noises are considered, and 2) Scenario II: the model uncertainty, time-varying actuator gain and bias faults, and measurement noises are involved.

The control parameters are selected as: $k_{1}=3, k_{2}=4$, $r_{1}=\frac{1}{3}, r_{2}=\frac{1}{2}, \eta=0.1$, and $\Phi=0.15$. In the adaptive laws: $\gamma_{1}=\frac{1}{40}, \gamma_{2}=\frac{1}{15}$, and $\gamma_{3}=\frac{1}{10} . \hat{c}_{1}(0)=\hat{c}_{2}(0)=\hat{c}_{3}(0)=0$.

For quantitatively evaluating the tracking performance, an index is defined as:

$$
\sigma_{p, j}=\sqrt{\frac{1}{t_{2}-t_{1}} \int_{t_{1}}^{t_{2}}\left|\sigma_{j}\right|^{2} d \tau}, \quad j=\mu, \alpha, \beta,
$$

where $\left[t_{1}, t_{2}\right]$ covers the time frame of the overall simulation. The defined metric is the scalar valued $L_{2}$ norm, as a measure of average tracking performance.

\section{B. Simulation Analysis of Scenario I}

It is highlighted in Fig. 2(a) that after the actuator faults take place $(t \geq 9 \mathrm{~s})$, the reference signal can be quickly tracked under the proposed FTC scheme. As can be seen from Fig. 2(b) and Fig. 2(c), the AOA and sideslip angle can converge to the intended values within finite time in the presence or absence of actuator faults. Focusing on Fig. 2, the developed FTC scheme allows the $\mathrm{HGV}$ to follow the prescribed tracking profiles as closely as possible, under the actuator faults and model uncertainties. The defined indices in Eq. (57) with respect to the bank angle, AOA, and sideslip angle are $0.4068^{\circ}, 0.1904^{\circ}$, and $0.1764^{\circ}$, respectively. Based on Fig. 3, the amplitude of the actuators becomes larger than that of the normal case, such that the effects induced by the faults can be eliminated. Key observations from Fig. 4 are: 1) the estimated values of the parameters $\left(\hat{c}_{1}, \hat{c}_{2}\right.$, and $\left.\hat{c}_{3}\right)$ hold at constant values to counteract model uncertainties $(0 \leq t<9 \mathrm{~s})$ and 2$)$ the estimated values respond appropriately by applying the adaptive laws after the occurrence of the actuator faults. 


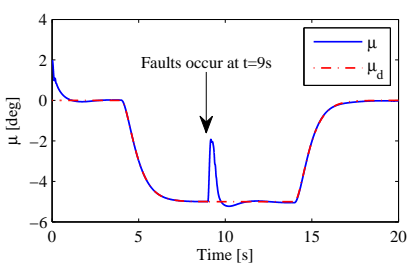

(a)

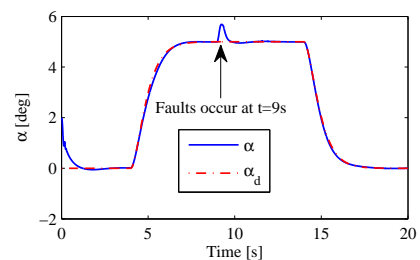

(b)

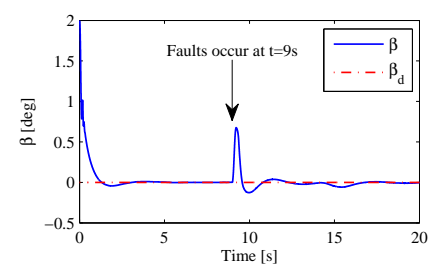

(c)

Fig. 2. The curves of the tracking angles in Scenario I.

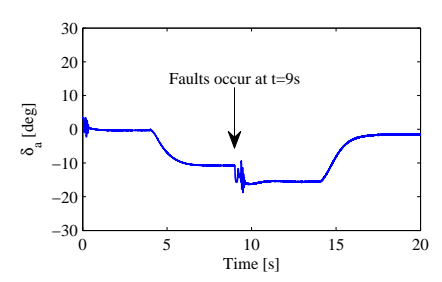

(a)

Fig. 3. The curves of the deflections in Scenario I.

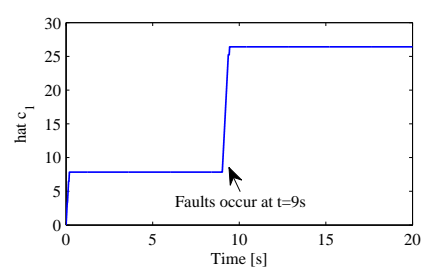

(a)

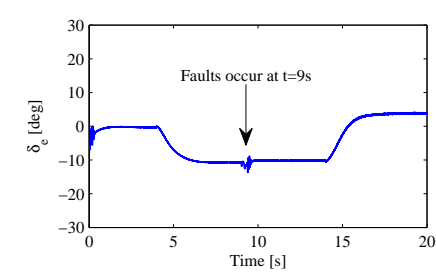

(b)

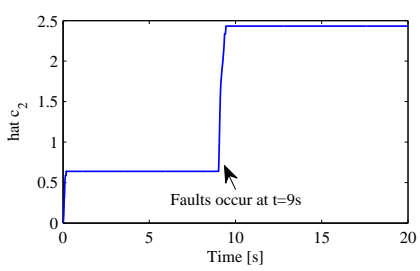

(b)

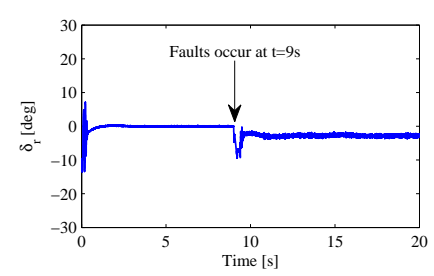

(c)

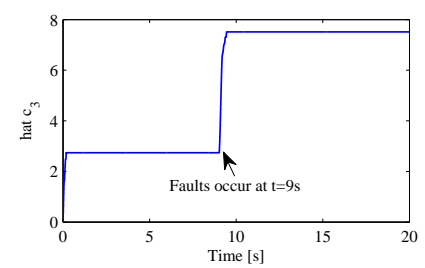

(c)

Fig. 4. The curves of the adaptive gains in Scenario I.

\section{Simulation Analysis of Scenario II}

The FTC performance against actuator time-varying faults is evaluated in Scenario II. From Fig. 5, the tracking performance is satisfactory, when the actuator time-varying malfunctions, measurement noises, and model uncertainties simultaneously exist. The HGV states can be steered to the intended values in a timely manner. The defined metrics corresponding to the bank angle, AOA, and sideslip angle are $0.4671^{\circ}, 0.2025^{\circ}$, and $0.2137^{\circ}$, respectively. As compared to those in Scenario I (see Table I), the performance is decreased by $14.82 \%$, $6.36 \%, 21.15 \%$, respectively. This condition arises due to that the impact of time-varying faults is worse than that of time-invariant ones. The deflections of the actuators and the adaptation process of the control gains are depicted in Fig. 6 and Fig. 7, respectively. The control gains can be promptly updated in response to the time-varying faults. The actuators are appropriately managed to maintain the HGV safety. In summary, the applicability of the developed FTC scheme is further verified through the simulation studies of Scenario II.

\section{CONCLUDING REMARKS}

An FTC architecture, including the multivariable integral TSMC and adaptive approaches suitable for HGV attitude tracking control, is developed against actuator faults and model uncertainties. The unique advantages of the proposed method
TABLE I

PERFORMANCE INDEX

\begin{tabular}{cccc}
\hline \hline- & $\sigma_{p, \mu}$ & $\sigma_{p, \alpha}$ & $\sigma_{p, \beta}$ \\
\hline Scenario I & 0.4068 & 0.1904 & 0.1764 \\
Scenario II & 0.4671 & 0.2025 & 0.2137 \\
\hline \hline
\end{tabular}

lie in three aspects. 1) The finite-time stability of the faulty HGV can be guaranteed so that unacceptable HGV behaviors are not created by actuator gain and bias malfunctions; 2) The composite-loop design under actuator faults is achieved on the basis of control-oriented model, without the need of the timescale separation principle; and 3) The multivariable integral TSMC method is presented to enable integration into the HGV FTC design, instead of the decoupled single-input and singleoutput method. The simulations of a full nonlinear model of the HGV dynamics show that the investigated scheme can be successfully employed to handle scenarios involving actuator faults and model uncertainties.

Despite that the proposed algorithm can be thought as a second order SMC, multivariable HOSMC with integration of dual-layer adaptive techniques, which can achieve a high level of accuracy and result in continuous control signals, is one of our future directions. 


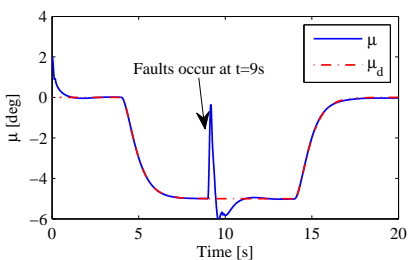

(a)

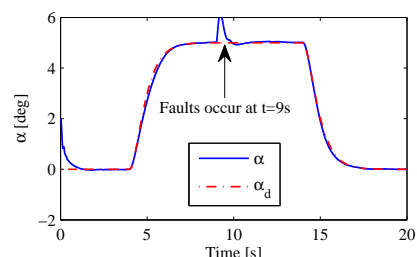

(b)

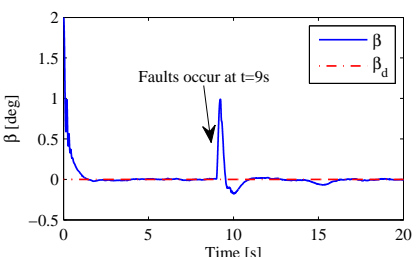

(c)

Fig. 5. The curves of the tracking angles in Scenario II.

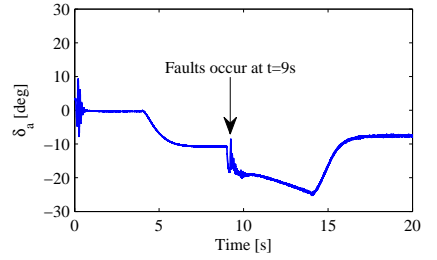

(a)

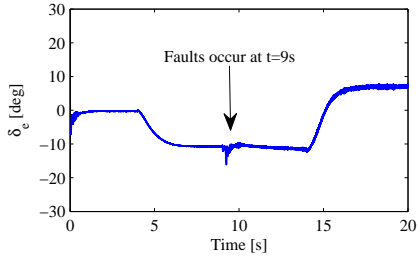

(b)

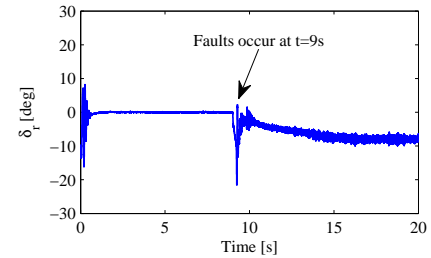

(c)

Fig. 6. The curves of the deflections in Scenario II.

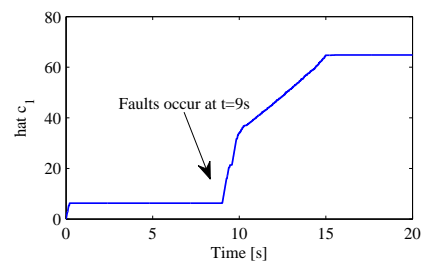

(a)

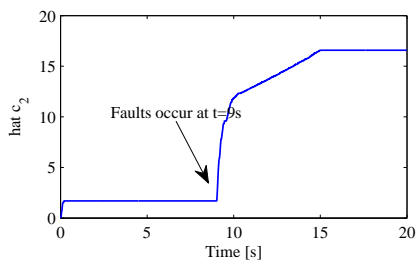

(b)

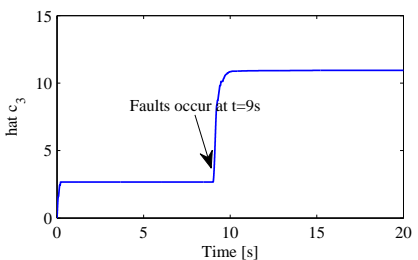

(c)

Fig. 7. The curves of the adaptive gains in Scenario II.

\section{ACKNOWLEDGEMENTS}

The authors would like to express their sincere gratitude to the Technical Editor and the anonymous reviewers whose insightful comments have helped to improve the quality of this paper considerably.

\section{REFERENCES}

[1] B. N. Pamadi, P. F. Covell, and P. V. Tartabini, "Aerodynamic Characteristics and Glide-Back Performance of Langley Glide-Back Booster," in Proc. 22nd Appl. Aerodyn. Conf. Exhib., Providence, USA, 2004, pp. 1-17.

[2] C. Michael, B. Peter, and K. Michael, "Adaptive Control for a Hypersonic Glider Using Parameter Feedback from System Identification," in Proc. AIAA Guid. Nav. Control Conf., Portland, USA, 2011, pp. 1-18.

[3] S. Banerjee, Z. J. Wang, B. Baur, F. Holzapfel, J. X. Che, and C. Y. Cao, " $L_{1}$ Adaptive Control Augmentation for the Longitudinal Dynamics of a Hypersonic Glider," J. Guid. Control Dyn., vol. 39, no. 2, pp. 275291, Feb. 2016.

[4] L. Fiorentini, A. Serrani, M. A. Bolender, and D. B. Doman, "Nonlinear Robust Adaptive Control of Flexible Air-Breathing Hypersonic Vehicles," J. Guid. Control Dyn., vol. 32, no. 2, pp. 401-416, Mar. 2009.

[5] L. G. Wu, X. Yang, and F. Li., "Nonfragile Output Tracking Control of Hypersonic Air-Breathing Vehicles with an LPV Model," IEEE/ASME Trans. Mechatronics, vol. 18, no. 4, pp. 1280-1228, Aug. 2013.

[6] H. J. Xu, M. D. Mirmirani, and P. A. Ioannou, "Adaptive Sliding Mode Control Design for a Hypersonic Flight Vehicle," J. Guid. Control Dyn., vol. 27, no. 5, pp. 829-838, Sep. 2004.

[7] J. Yang, Z. H. Zhao, S. H. Li, and W. X. Zheng, "Composite Predictive Flight Control for Airbreathing Hypersonic Vehicles," Int. J. Control, vol. 87, no. 9, pp. 1970-1984, Sep. 2014.
[8] L. Fiorentini and A. Serrani, "Adaptive Restricted Trajectory Tracking for a Non-Minimum Phase Hypersonic Vehicle Model," Automatica, vol. 48, no. 7, pp. 1248-1261, Jul. 2012.

[9] P. Yu and Y. Shtessel, "Continuous Higher Order Sliding Mode Control with Adaptation of Air Breathing Hypersonic Missile," Int. J. Adapt. Control Signal Process., vol. 30, no. 8-10, pp. 1099-1117, Aug.-Oct. 2016.

[10] Y. M. Zhang and J. Jiang, "Bibliographical Review on Reconfigurable Fault-Tolerant Control Systems," Annu. Rev. Control, vol. 32, no. 2, pp. 229-252, Dec. 2008.

[11] X. Yu and J. Jiang, "A Survey of Fault-Tolerant Controllers Based on Safety-Related Issues," Аnnu. Rev. Control, vol. 39, no. 1, pp. 46-57, Apr. 2015.

[12] Q. K. Shen, B. Jiang, and V. Cocquempot, "Fault-Tolerant Control for T- Fuzzy Systems with Application to Near-Space Hypersonic Vehicle with Actuator Faults," IEEE Trans. Fuzzy Syst., vol. 20, no. 4, pp. 652-665, Aug. 2012.

[13] Q. K. Shen, B. Jiang, and V. Cocquempot, "Fuzzy Logic SystemBased Adaptive Fault-Tolerant Control for Near-Space Vehicle Attitude Dynamics with Actuator Faults," IEEE Trans. Fuzzy Syst., vol. 21, no. 2, pp. 289-300, Apr. 2013.

[14] F. Wu and X. Cai, "Switching Fault Tolerant Control of a Flexible Air-Breathing Hypersonic Vehicle," Proc. Inst. Mech. Eng. I J. Syst. Control Eng., vol. 227, no. 1, pp. 24-38, Jan. 2013.

[15] X. X. Hu, H. R. Karimi, L. G. Wu, and Y. Guo, "Model Predictive Control-Based Nonlinear Fault Tolerant Control for Air-Breathing Hypersonic Vehicles," IET Control Theory Appl., vol. 8, no. 13, pp. 1147-1153, Sep. 2014.

[16] J. Zhao, B. Jiang, P. Shi, Z. F. Gao, and D. Z. Xu, "Fault-Tolerant Control Design for Near-Space Vehicles Based on a Dynamic Terminal Sliding Mode Techniques," Proc. Inst. Mech. Eng. I J. Syst. Control Eng., vol. 226, no. 6, pp. 787-794, June 2012.

[17] Y. H. Ji, H. L. Zhou, and Q. Zong, "Adaptive Active Fault-Tolerant 
Control of Generic Hypersonic Flight Vehicles," Proc. Inst. Mech. Eng. I J. Syst. Control Eng., vol. 229, no. 2, pp. 130-138, Feb. 2015.

[18] H. An, J. X. Liu, C. H. Wang, and L. G. Wu, "Approximate BackStepping Fault-Tolerant Control of the Flexible Air-Breathing Hypersonic Vehicle," IEEE/ASME Trans. Mechatronics, vol. 21, no. 3, pp. 1680-1691, June 2016.

[19] Z. H. Man, A. P. Paplinski, and H. R. Wu, "A Robust MIMO Terminal Sliding Mode Control Scheme for Rigid Robotic Manipulator," IEEE Trans. Autom. Control, vol. 39, no. 12, pp. 2464-2469, Dec. 1994.

[20] J. Zheng, H. Wang, Z. H. Man, J. Jin, and M. Y. Fu, "Robust Motion Control of Linear Motor Positioner Using Fast Nonsingular Terminal Sliding Mode," IEEE/ASME Trans. Mechatronics, vol. 20, no. 4, pp. 1743-1752, Aug. 2015.

[21] M. Jin, J. Lee, and K. Ahn, "Contiuous Nonsingular Terminal SlidingMode Control of Shape Memory Alloy Actuators Using Time Delay Estimation," IEEE/ASME Trans. Mechatronics, vol. 20, no. 2, pp. 899909, Apr. 2015.

[22] Z. F. Gao, B. Jiang, P. Shi, M. S. Qian, and J. X. Lin, "Active Fault Tolerant Control Design for Reusable Launch Vehicle Using Adaptive Sliding Mode Technique," J. Franklin Inst., vol. 349, no. 4, pp. 1543 1560, May 2012.

[23] H. B. Sun, S. H. Li, and C. Y. Sun, "Robust Adaptive Integral-SlidingMode Fault-Tolerant Control for Airbreathing Hypersonic Vehicles," Proc. Inst. Mech. Eng. I J. Syst. Control Eng., vol. 226, no. 10, pp. 1344-1355, Oct. 2012.

[24] T. Cao, Z. F. Gao, M. S. Qian, and J. Zhao, "Passive Fault Tolerant Control Approach for Hypersonic Vehicle with Actuator Loss of Effectiveness Faults," in Proc. 28th Chi. Control Decision Conf., Yinchuan, China, 2016, pp. 5951-5956.

[25] Y. Shtessel, J. Buffington, and S. Banda, "Multiple Timescale Flight Control Using Reconfigurable Sliding Modes," J. Guid. Control Dyn., vol. 22 , no. 6, pp. 873-883, Nov. 1999.

[26] B. L. Tian, W. R. Fan, R. Su, and Q. Zong, "Real-Time Trajectory and Attitude Coordination Control for Reusable Launch Vehicle in Reentry Phase," IEEE Trans. Ind. Electron., vol. 62, no. 3, pp. 1639-1650, Mar. 2015

[27] S. Bhat and D. Bernstein, "Finite-Time Stability of Continuous Autonomous Systems," SIAM J. Control Optim., vol. 38, no. 3, pp. 751766, June 2000.

[28] X. Yu and J. Jiang, "Hybrid Fault-Tolerant Flight Control System Design Against Partial Actuator Failures," IEEE Trans. Control Syst. Technol., vol. 20, no. 4, pp. 871-886, Jul. 2012.

[29] B. Xiao and S. Yin, "Velocity-free Fault Tolerant and Uncertainty Attentuation Control for a Class of Nonlinear Systems," IEEE Trans. Ind. Electron., vol. 63, no. 7, pp. 4400-4411, Jul. 2016.

[30] A. Levant, "Higher-Order Sliding Modes, Differentiation and OutputFeedback Control," Int. J. Control, vol. 76, no. 9, pp. 924-941, Sep. 2003.

[31] Y. Shtessel, C. Edwards, L. Fridman, and A. Levant, Sliding Mode Control and Observation. London, UK: Spinger, 2013.

[32] R. Su, Q. Zong, B. L. Tian, and M. You, "Comprehensive Design of Disturbance Observer and Non-Singular Terminal Sliding Mode Control for Resuable Launch Vehicles," IET Control Theory Appl., vol. 9, no. 12, pp. 1821-1830, Dec. 2015.

[33] L. Wang, T. Y. Chai, and L. Zhai, "Neural-Network-Based Terminal Sliding Mode Control of Robotic Manipulators Including Actuator Dynamics," IEEE Trans. Ind. Electron., vol. 56, no. 9, pp. 3294-3304, Sep. 2009

[34] Y. Feng, X. Yu and Z. Man, "Nonsingular Terminal Sliding Mode Control of Rigid Manipulators," Automatica, vol. 38, no. 12, pp. 21572167, Dec. 2002.

[35] Y. Feng, X. Yu, and F. Han, "On Nonsingular Terminal Sliding-Mode Control of Nonlinear Systems," Automatica, vol. 49, no. 6, pp. 17151722, June 2013.

[36] V. I. Utkin, Sliding Modes in Control and Optimization. Berlin, Germany: Springer, 1992.

[37] H. Alwi and C. Edwards, "Fault Detection and Fault-Tolerant Control of a Civil Aircraft Using a Sliding-Mode-based Scheme", IEEE Trans. Control Syst. Technol., vol. 16, no. 3, pp. 499-510, May 2008.

[38] Q. Shen, D. Wang, S. Zhu, and E. K. Poh, "Inertia-Free Fault-Tolerant Spacecraft Attitude Tracking Using Control Allocation", Automatica, vol. 62, pp. 114-121, Dec. 2015

[39] Y. Shtessel, M. Taleb, and F. Plestan, "A Novel Adaptive-Gain Supertwisting Sliding Mode Controller: Methodology and Application," Automatica, vol. 48, no. 5, pp. 759-769, May 2012.

[40] C. Edwards and Y. Shtessel, "Dual-Layer Adaptive Sliding Mode Control," in Proc. IEEE Conf. Decision Control, Portland, USA, 2014, pp. $4524-4529$.

[41] C. Edwards and Y. Shtessel, "Adaptive Continuous Higher Order Sliding Mode Control," Automatica, vol. 65, pp. 183-190, Mar. 2016.

[42] C. Edwards and Y. Shtessel, "Adaptive Dual Layer Super-Twisting Control and Observation," Int. J. Control, vol. 89, no. 9, pp. 17591766, Sep. 2016.

[43] I. Nagesh and C. Edwards, "A Multivariable Super-Twisting Sliding Mode Approach," Automatica, vol. 50, no. 3, pp. 984-988, Mar. 2014.

[44] B. L. Tian, L. P. Yin, and H. Wang. "Finite-Time Reentry Attitude Control based on Adaptive Multivariable Disturbance Compensation,"IEEE Trans. Ind. Electron., vol. 62, no. 9, pp. 5889-5898, Sep. 2015.

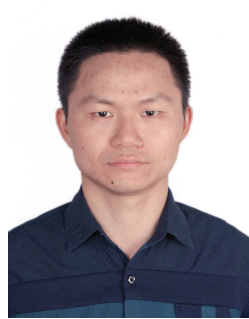

Peng Li received the B.S. degree from Wuhan University, Wuhan, China, in 2004, and the M.S., and $\mathrm{Ph} . \mathrm{D}$. degrees from National University of Defense Technology, Changsha, China, in 2006 and 2011, respectively. $\mathrm{He}$ is a Lecturer with the College of Mechatronics Engineering and Automation, National University of Defense Technology, and also with the State Key Laboratory of High Performance Computing in the same university. His current research interests include high order sliding-mode control and fault-tolerant flight control.

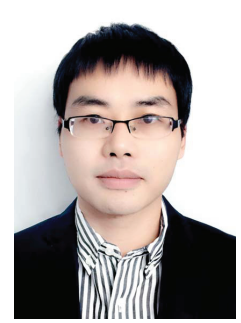

Xiang Yu (M'10-SM'13) received the B.S., M.S., and Ph.D. degrees from Northwestern Polytechnical University, Xi' an, China, in 2003, 2004, and 2008, respectively. From 2009 to 2013, he was a postdoctoral research fellow at The University of Western Ontario, London, ON, Canada. He has been working at Concordia University since 2014. His main research interests include GNC for aerospace engineering systems, and fault-tolerant control.

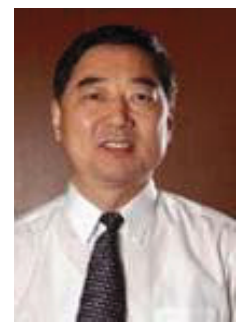

Youmin Zhang (M'99-SM'07)received the B.S., M.S., and Ph.D. degrees from Northwestern Polytechnical University, Xian, China, in 1983, 1986, and 1995 , respectively. Dr. Zhang is currently a Professor with the Department of Mechanical, Industrial \& Aerospace Engineering, Concordia University. His current research interests include fault diagnosis and fault-tolerant (flight) control systems, cooperative GNC of unmanned aerial/space/ground/surface vehicles. He has authored four books, over 450 journal and conference papers, and book chapters. Dr. Zhang is a Fellow of CSME, a Senior Member of AIAA and IEEE, Vice-President of International Society of Intelligent Unmanned Systems, and a member of the Technical Committee for several scientific societies. He is an Editorial Board Member, Editor-in-Chief, Editor-at-Large, Editor or Associate Editor of several international journals. He has served as the General Chair, the Program Chair, and IPC Member of several international conferences.

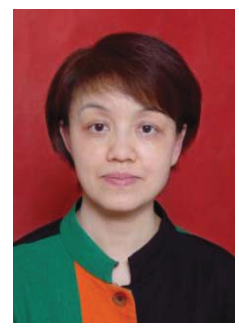

Xiaoyan Peng is currently a Professor at the College of Mechanical and Vehicle Engineering, $\mathrm{Hu}$ nan University. Her research interests cover control of mechatronic systems and safety analysis of autonomous vehicles. 